\title{
SEMA4D upregulation signals neuronal stress and triggers reactive transformation of astrocytes
}

\section{Maurice Zauderer ( $\nabla$ mzauderer@vaccinex.com )}

Vaccinex (United States)

Vikas Mishra

Vaccinex

Crystal Mallow

Vaccinex

Elaine Gersz

Alisha Reader

Vaccinex

Leslie Balch

Vaccinex

David Oakes

University of Rochester

Alan Howell

Vaccinex

Ernest S. Smith

Vaccinex

Terrence L. Fisher

Vaccinex

Elizabeth E. Evans

Vaccinex

\section{Andrew Feigin}

New York University Langone Health, and The Marlene and Paolo Fresco Institute for Parkinson's and Movement Disorders

\section{Article}

Keywords: SEMA4D, SEMA4D+, FDG-PET, AD, pepinemab

Posted Date: January 19th, 2021

DOI: https://doi.org/10.21203/rs.3.rs-62001/v1 
License: (c) (i) This work is licensed under a Creative Commons Attribution 4.0 International License. Read Full License 
SEMA4D upregulation signals neuronal stress and triggers reactive transformation of astrocytes

Maurice Zauderer*, Vikas Mishra, Crystal Mallow, Elaine Gersz, Leslie Balch, Alan Howell, Ernest S.

Smith, Terrence L. Fisher, Elizabeth E. Evans

*Corresponding author: Dr. Maurice Zauderer, Vaccinex, Inc., 1895 Mt. Hope Avenue, Rochester, NY, USA; mzauderer@vaccinex.com; (585) 271-2827.

Vaccinex, Inc., Research, Rochester, New York, USA 


\section{ABSTRACT}

2 The close interaction and interdependence of glial cells and neurons allows for the possibility that glial

3 dysfunction contributes to and amplifies neurodegenerative pathology. Drivers of glial cell activation

4 may represent important targets to preserve normal homeostatic maintenance and modify disease

5 progression. We report here that semaphorin 4D (SEMA4D) is a trigger of astrocyte activation and is

6 upregulated in neurons under stress or damage, as characterized in brains from mouse models as well as

7 patients with Huntington's and Alzheimer's disease. These SEMA4D+ neurons are in close proximity to

8 reactive astrocytes that evidence distinctive morphologic changes and downregulation of glutamine

9 synthetase associated with reactive astrogliosis along with loss of some key normal astrocyte functions,

10 including glucose transport and glutamate recycling. It is further demonstrated that astrocytes express

11 cognate plexin receptors for SEMA4D and that binding to the ligand results in collapse of the actin

12 cytoskeleton and down regulation of glutamine synthetase, and glucose and glutamate transport. These

13 effects are prevented and reversed in cultures of iPSC-derived human astrocytes by antibody blockade of

14 SEMA4D. In vivo antibody neutralization of SEMA4D reduced reactive astrocyte phenotype and

15 prevented characteristic loss of GABAergic synapses in brains of CVN mice. These results suggest that

16 SEMA4D represents a novel driver of astrocyte transformation that is upregulated early during disease

17 progression and that antibody blockade of SEMA4D can preserve normal astrocyte and neuronal

18 function.

20 Introduction

21 Glial cells are increasingly recognized for their important contribution to onset and progression of

22 neurodegenerative diseases ${ }^{1}$. Studies from multiomic, epidemiologic, clinical and animal models have

23 advanced the understanding of interactions between neurons and glial cells and the role of

24 neuroinflammatory mechanisms. Astrocytes in particular have been increasingly recognized as key 
contributors to pathology and progression of AD, HD, PD, ALS and other slowly progressive neurodegenerative diseases. Astrocytes play a critical role in the integration of neural synaptic networks and are well-positioned to couple energy metabolism with synaptic activity. Fine cytoplasmic processes of astrocytes cradle synapses and express glutamate receptors while astrocyte endfeet express glucose transporter and completely cover brain capillaries. Astrocytes are extremely responsive to disruptions in their microenvironment, as occurs in injury and neurodegenerative disorders, with morphological and functional changes referred to as reactive astrogliosis and astrocytopathy ${ }^{2}$. Such astrocytic transformation may be a key pathological driver of disease, as reactive astrogliosis has been shown to precede neuronal loss and to be sufficient to trigger disease phenotype in animal models ${ }^{3}$. Astrogliosis is characterized by gain of inflammatory processes ${ }^{4}$, as well as loss of normal homeostatic functions. Dysregulation of normal functions include impairment in glucose uptake and neurotransmitter recycling due to downregulation of key receptors and enzymes in the respective pathways, including Glut1, MCT4, and glycolytic enzymes that regulate metabolic activity ${ }^{5,6}$ and EAAT2 and glutamine synthetase (GS) required for recycling glutamate and GABA transmitters ${ }^{7}$. Cytoskeletal rearrangements are another hallmark of astrogliosis characterized by upregulation of glial fibrillary acidic protein (GFAP) and hypertrophic cell bodies with retracted short processes and loss of fine processes ${ }^{8}$. These morphologic changes may influence astroglial pathogenesis via disruption of normal functions that depend on cytoplasmic projections, including neuro-glial and glial-endothelial networks.

Semaphorin 4D (SEMA4D) regulates the actin cytoskeleton through small membrane Rho GTPases ${ }^{9-11}$. The actin cytoskeleton controls cell morphology and the ability to extend projections required for cell migration and for direct interactions with other cells. In the CNS, SEMA4D signals through its high affinity plexin receptors (PLXNB1, PLXNB2) (i) to activate glial cells ${ }^{12,13}$, (ii) to inhibit migration and differentiation of glial progenitor cells that can replace damaged oligodendrocytes and replenish 
$\operatorname{astrocytes}^{9,10,14,15}$, and (iii) to disrupt endothelial tight junctions that are required for the integrity of the

50 blood-brain barrier $(\mathrm{BBB})^{12}$.

51 We report here that neurons upregulate SEMA4D during underlying Huntington's and Alzheimer's

52 disease progression and that this is associated with inflammatory transformation of astrocytes as

53 reflected in altered morphology and downregulation of GS expression. Glutamine synthetase is

54 predominantly expressed by normal astrocytes in the brain and is a key enzyme in recycling glutamate

55 and GABA neurotransmitters. Upregulation of SEMA4D in neurons was detected in brain sections of an

56 HD transgenic model (Q175) as well as in staged HD patient brain autopsy samples. Similarly,

57 upregulated SEMA4D was detected in AD brain autopsy sections and in CVN-AD mice. Astrocytes are

58 shown to express the high affinity plexin-B1 receptor for SEMA4D and interaction with the ligand

59

60

61

62

63

64

65

66

67

68

69

70

71

72

triggers reversible depolymerization of F-actin and collapse of the cytoskeleton. A number of

observations of our own and others further suggest that SEMA4D/plexin-B1 signaling affects the ability of mature astrocytes to extend cytoplasmic projections required for their normal functions including glucose transport through direct interaction with brain capillaries ${ }^{16-19}$ and glutamate recycling through projections that cradle synapses. Therefore, blockade of SEMA4D signaling may serve to normalize astrocytic and neuronal functions and slow neurodegenerative disease progression. We previously reported that an anti-SEMA4D antibody ameliorated neurodegenerative processes in YAC128 HD transgenic mice ${ }^{20}$.

Here, we further elucidate mechanism of action and demonstrate that antibody blockade prevented or reversed SEMA4D-induced changes to astrocyte morphology and function in vitro and in vivo. In the CVN mouse model of $\mathrm{AD}$, treatment with anti-SEMA4D antibody restored normal astrocyte morphology and reversed neuronal dysfunction, as evidenced by preventing characteristic loss of

1 GABAergic synapses. We will report elsewhere results of a phase 2 study that indicates clinical benefit of SEMA4D blockade in Huntington's disease. 
RESULTS:

\section{Upregulation of SEMA4D in neurons during Huntington's and Alzheimer's disease progression}

As in the case of other slowly progressive neuroinflammatory/neurodegenerative diseases, chronic activation of inflammatory glial cells is believed to play a key role in the underlying progression of Huntington's disease ${ }^{3,21-24}$. We have previously reported that SEMA4D triggers depolymerization of the actin cytoskeleton in glial progenitor cells and impedes their migration ${ }^{12}$. Similar effects on the cytoskeleton of mature astrocytes are characteristic of astrogliosis and could profoundly impact their normal function. Additionally, we previously reported that treatment with anti-SEMA4D antibody ameliorates neurodegenerative processes in YAC128 HD transgenic mice ${ }^{20}$. We, therefore, investigated expression of SEMA4D in neural tissue during HD progression and potential expression of cognate receptors on astrocytes. SEMA4D is here shown to be upregulated in neurons of HD transgenic Q175 mice (Fig. 1a, b), increasing with age as disease progresses. Upregulation of SEMA4D can be detected even at 3 months of age (Fig. 1a, b), prior to disease symptom onset, which typically occurs $\sim 5$ months of age in this mouse model. A significant reduction in the number of $\mathrm{NeuN}+$ neurons at 9 months of age provides evidence of neuronal loss with increasing SEMA4D expression and disease progression. To determine whether similar changes in expression of neuronal SEMA4D characterizes human disease progression, we examined autopsy samples obtained from the NIH brain bank of 3 subjects each representative of normal control, and HD pathological stage 0 , stage 1 , and stage 2 . Staining for SEMA4D and the neuronal marker $\mathrm{HuC} / \mathrm{HuD}$ (Fig. 1c) shows statistically significant upregulation of SEMA4D in neurons (Fig. 1d) and evidence of neuronal loss (Fig. 1e) in frontal and parietal lobes, and in the caudoputamen striatal region. Early neuronal loss, especially of medium spiny neurons in the striatum with increasing involvement of the cortical regions as the disease progresses is characteristic of 
$\mathrm{HD}^{25}$. SEMA4D levels in $\mathrm{HuC} / \mathrm{HuD}+$ neurons continue to increase with increasing pathological stage,

97 98

99 100 101 102 103 104 105 106 perhaps indicating increasing neuronal stress during disease progression.

To determine if SEMA4D upregulation is a common underlying pathogenic mechanism in another neurodegenerative disease, we evaluated autopsy sections from subjects with AD. Consistent with observations in HD, we observed significant upregulation of SEMA4D and a reduction in the neuronal density in numerous regions of interest in $\mathrm{AD}$ brains compared to normal controls. Significant changes in the frontal cortex, temporal lobe and thalamus of AD affected subjects are shown in Figure 2.

\section{Reactive astrogliosis during disease progression}

Reactive astrocytes ${ }^{26,27}$ undergo morphological and functional changes, including significant changes in gene expression ${ }^{7,28}$. To characterize and quantify complex morphological changes in heterogeneous astrocytes, scale-invariant parameters like fractal dimensions (Df) ${ }^{29,30}$ can be employed. Astrocytes in HD transgenic Q175 mice (Fig. 3a) and autopsy tissue from HD and AD subjects (Fig. 3b) display characteristic high GFAP immunoreactivity, enlarged cell soma, and significant increase in Df values in the frontal cortex compared to astrocytes in normal healthy brains. Significant reduction of GS expression starting at HD stage 0 indicates the presence of reactive astrocytes ${ }^{7}$ early in human disease

(Fig 4). Furthermore, the close proximity of GS-positive endfeet of stained astrocytes (purple) to $\mathrm{HuC} / \mathrm{HuD}$-positive neurons (green or yellow with SEMA4D co-stain) observed in normal healthy brain tissue appears to be disrupted in the HD brain (Fig. 4a). Consistent with observations in HD subjects, we observed significant reduction in GS expression and morphologic changes associated with reactive astrogliosis in numerous regions of interest in AD brains as well (Fig 4c). Significant changes in the frontal cortex, temporal lobe and thalamus of $\mathrm{AD}$ affected subjects compared to normal controls are shown in Figure 4d. Collectively, these morphological changes and downregulation of GS are 
consistent with a reactive astrocyte phenotype and coincident with disease progression and increasing upregulation of SEMA4D in neurons.

\section{Astrocytes express plexin-B1 receptors for SEMA4D and ligand triggers morphological} transformation and reduced metabolic function.

To determine whether SEMA4D upregulation in neurons can trigger astrocyte transformation, we investigated whether astrocytes express plexin-B1 receptors and whether binding of SEMA4D impacts morphologic and functional changes associated with astrogliosis. As shown in Figure 5a, plexin-B1 receptors are highly expressed in purified astrocytes, and recombinant SEMA4D rapidly triggers approximately $60 \%$ depolymerization of F-actin after only one hour of exposure in vitro. As would be expected, addition of SEMA4D reduces the amount of astrocyte cytoplasmic projections that spontaneously extend in culture which is, however, reversible and recovers following addition of SEMA4D blocking antibody at $20 \mathrm{hrs}$ (Fig. 5b). Similarly, SEMA4D induced morphologic changes in primary human astrocyte cultures, as evidenced by shortened length and reduced number of primary neurite branches (Fig. 5c). These effects were significantly inhibited by addition of blocking antibody to SEMA4D. Extensive astrocytic branching facilitates interactions with neuronal junctions and brain capillaries and is, therefore, critical to astrocyte function.

Glutamate recycling and glucose transport are important normal functions of astrocytes and may afford the opportunity to couple synaptic activity with brain energy metabolism. Both functions are significantly disrupted upon reactive astrocyte transformation ${ }^{6,31}$. Excitatory amino acid transporter (EAAT)-2 is one of the major astrocytic glutamate transporters, regulating glutamate uptake at the synaptic cleft in order to maintain homeostasis and potentially prevent adventitious signaling and excitotoxic activity. Binding of rSEMA4D to PLXNB1 receptor expressed on primary human astrocytes significantly reduced expression of EAAT-2 glutamate transporter (Fig. 6a). As expected, antiSEMA4D antibody (VX15) alone has no effect on EAAT-2 expression. In contrast, blocking antibody 
(VX15) significantly inhibited effects of SEMA4D and restored EAAT-2 to near control levels. To determine effects of SEMA4D on astrocyte energy metabolism, expression of glucose transporter (GLUT-1), necessary for glucose uptake by astrocytes, and monocarboxylate transporter (MCT) 4, the astrocytic transporter of lactate for diffusion to neurons, were evaluated. Significant reduction in expression of GLUT-1 and MCT4 on astrocytes was observed following incubation with recombinant SEMA4D (Fig. 6b). As expected, anti-SEMA4D antibody (VX15) alone has no effect on GLUT-1 and MCT4 expression, while blocking antibody (VX15) significantly inhibited effects of SEMA4D binding to PLXNB1 receptor on astrocytes.

To further demonstrate effects of SEMA4D signaling on astrocytic functional activity, we assessed the ability of astrocytes to transport glucose in presence of SEMA4D (Fig. 6c). SEMA4D reduced glucose uptake in cultured astrocytes by 24 hours, while SEMA4D antibody (VX15) alone had no effect. However, in the presence of rSEMA4D, VX15 blocked binding to inhibit the reduction of glucose uptake by astrocytes. Addition of SEMA4D antibody 24 hours after rSEMA4D treatment reversed the SEMA4D-induced conversion of astrocytes to hypometabolic state.

\section{SEMA4D regulation of astrogliosis impacts neuronal loss in mouse model of AD.}

Based on these results, we surmised that SEMA4D upregulation during disease progression could activate astrocytes and degrade their ability to perform normal functions including glucose metabolism and neurotransmitter recycling (Fig. 6d). To assess the therapeutic potential of blocking SEMA4D to restore these functions, SEMA4D antibody treatment was evaluated in the CVN mouse model of AD, which displays characteristics of AD progression, including reactive gliosis, amyloid deposition, phosphorylated tau protein, spatial memory impairments, and significant neuronal death in hippocampal, cortical, and thalamic regions ${ }^{32-34}$. Mice were treated weekly starting at 26 weeks of age, around the time of symptom onset, and brains were collected at 41 weeks of age, when major pathologic changes including glial activation and neuronal degeneration have occurred. SEMA4D upregulation (data not 
168

169

shown) and astrocyte morphologic transformation was confirmed in the CVN mice (Fig 7). Treatment with SEMA4D blocking antibody preserved normal morphology (Fig. 7 a, b) in hippocampal astrocytes of CVN mice. Astrocytic glutamine synthetase expression was significantly reduced in the hippocampus of CVN mice compared to wild type mice $(\mathrm{p}=0.0054)$, however no significant difference was observed in CVN mice treated with SEMA4D blocking antibody compared to wild type controls $(\mathrm{p}=0.9276)$. It has been reported that disruption of the GS-dependent conversion of glutamate to glutamine cycle in reactive astrocytes has a differential effect on loss of inhibitory GABAergic relative to excitatory glutamatergic signaling, It was suggested that this may be due to differences in transmitter pools at inhibitory versus excitatory synapses or alternative sources of glutamine for glutamate synthesis ${ }^{7}$. Dysfunction of inhibitory synapses was observed in CVN mice, as evidenced by loss of somatostatin and neuropeptide Y (NPY), markers of inhibitory synapses in the interneurons of the subiculum and dentate gyrus. No effects on excitatory or total synapses were observed in diseased mice, as determined by vesicular glutamate transporter 1 (VGLUT-1) and synaptophysin expression (Fig 7c). These results demonstrate that SEMA4D blockade prevented the differential loss of inhibitory synapses seen in CVN mice that could be an important factor in disrupting neural circuits.

\section{DISCUSSION:}

Although the clinical manifestations of HD and AD clearly indicate neuronal deficits, the close interaction and interdependence of glial cells and neurons allows for the possibility of a glial contribution to neuronal pathology by initiating and/or amplifying neuronal dysfunction. Astroglial transformation in response to neuronal stress creates a disease-permissive landscape that contributes to neuronal malfunction, neuronal death and neurological deficits ${ }^{3,23,24,35,36}$. In the case of HD, neuronal stress may be the result of accumulation of mutant huntingtin protein aggregates. We speculate that 
neuronal stress in other slowly progressive neuroinflammatory and neurodegenerative diseases such as

Alzheimer's may follow from a different physiological insult but result in similar inflammatory and pathologic response. Expression of mutant huntingtin in glial cells has, for example, been reported to contribute to neuronal excitotoxicity ${ }^{21}$. Importantly, reconstitution of the CD44+ astrocyte lineage in wild-type mice with precursors derived from embryonic glial progenitor cells or from induced pluripotent stem cells isolated from HD patients has been shown to be sufficient to reproduce many aspects of the HD phenotype; conversely, reconstitution of HD transgenic mice with normal human astrocytes is reported to ameliorate features of the disease phenotype ${ }^{3}$. Furthermore, disease associated upregulation of genes related to neuroinflammation and astrocyte activation, reveal important astrocyte contributions to the pathogenesis and progression of $\mathrm{HD}^{37,38}, \mathrm{AD}^{39,40}$ and other neurodegenerative diseases ${ }^{8}$.

What mechanism(s) give rise to disease associated pathogenic changes in astrocytes? We report here that during underlying disease progression in both HD and AD transgenic mice and patients, SEMA4D is upregulated in neurons (Fig. 1,2). We further demonstrate that astrocytes express high affinity plexinB1 receptors for SEMA4D (Fig. 5a). Binding of recombinant SEMA4D to purified GFAP+ astrocytes in vitro triggers significant depolymerization of F-actin (Fig. 5a) and restricts the ability to extend cytoplasmic projections which is, however, reversible and can be recovered following addition of SEMA4D blocking antibody (Fig. 5b, 5c). These results suggest that in the presence of SEMA4D the ability of astrocytes to perform normal functions that depend on cytoplasmic projections, for example, interaction with brain capillaries to facilitate glucose transport and perisynaptic cytoplasmic projections that express glutamate receptors responsible for recycling $80 \%$ of free glutamate, would be degraded. Indeed, we observed rapid morphologic changes within one hour of in vitro treatment with SEMA4D, while functional effects of SEMA4D on dendritic extension are observed at 10-24 hours. Concurrent 
with these morphologic changes, we observed downregulation of astrocyte glucose transporter GLUT-1

217 and lactate transporter MCT4 at 24-48 hours. Importantly, these changes perturbed normal astrocytic

218 function, as evidenced by downregulation of glucose uptake, which was reversible with antibody

219 blockade of SEMA4D (Fig. 6). Glutamate transport was also regulated by SEMA4D, as evidenced by

220 reduced expression of astrocytic glutamate transporter EAAT-2. A model, therefore, emerges in which

221 (i) SEMA4D is upregulated as a signal of stress at sites of neuronal injury; (ii) this triggers inflammatory

222 activation and morphologic changes of plexin-B1/B2 positive astrocytes in close proximity to neurons;

223 and (iii) compromises the normal role of astrocytes in glucose transport and glutamate recycling and

224 may result in other functional deficits. A corollary is that blocking SEMA4D signaling could prevent or

225 reduce disease-associated inflammatory transformation and loss of normal astrocyte functions during

226 neuroinflammatory disease progression.

The normal astrocytic functions of glucose transport and glutamate recycling are critical to neuronal

function ${ }^{6,31}$. Dysfunction in these circuits are often the initiating event or part of the cascade leading to neurological disorders and neurodegeneration. In an analysis of protein networks, sugar metabolism emerged as one of the most significantly associated modules to AD pathology and cognitive impairment and was enriched among activated glia ${ }^{41}$. A consequence of altered glucose and glutamate transport is maintenance of brain ionic equilibrium within astrocytes and their ability to efficiently convert glutamate to glutamine ${ }^{42}$, collectively regulating neuroprotective effects and synaptic function.

235 Glutamine synthetase is a key enzyme in glutamate recycling and is predominantly expressed by 236 astrocytes in the brain. Downregulation of GS has been previously described as a marker of 237 inflammatory activation of astrocytes ${ }^{7,28}$. To determine whether reactive transformation of astrocytes is 238 a feature of disease progression, we examined glutamine synthetase (GS) expression in GFAP+ cells and 239 determined that there is striking reduction in GS expression with advancing disease (Fig. 4). We 
confirmed that this was accompanied by morphological changes associated with reactive astrogliosis in HD and AD (Fig. 3, 4). Inhibitory synapses are especially vulnerable to changes in glutamine levels ${ }^{7}$ and are also known to be critically affected in AD. NPY and somatostatin, produced mainly by GABAergic interneurons, are associated with cognitive and emotional processes and appear to play an important role in learning and memory ${ }^{43,44}$. Hippocampal NPY+ neurons are strongly affected in early stages of AD pathology and significantly reduced in brains of AD patients ${ }^{45}$ and CVN mice ${ }^{46}$. In the CVN model, treatment with SEMA4D blocking antibodies prevented transformation to the astrocytic reactive phenotype, restored expression of GS responsible for recycling glutamine stores to the neurons, and thereby inhibited the loss of inhibitory NPY+ and somatostatin+ neurons (Fig. 7).

Here, we provide evidence of SEMA4D regulation of astrocytic function, but we also consider the possibility that SEMA4D effects on other glia may contribute to neuroprotective benefits observed in $\mathrm{HD}^{20}$ and AD mice treated with SEMA4D antibodies. We previously reported that SEMA4D blocking antibody prevents inflammatory activation of murine Iba-1+ microglia at the site of demyelinated lesions in spinal cord ${ }^{12}$. We also note that additional mechanisms may contribute to the effect of SEMA4D on energy metabolism. We have previously reported that SEMA4D inhibits the migration and differentiation of glial progenitor cells that are able to replenish astrocytes and repair demyelinated lesions ${ }^{12}$. Blocking SEMA4D could, therefore, also change the balance between normal and inflammatory glia as well as between normal and compromised myelinated neurons. Other reported effects of blocking SEMA4D include protecting the integrity of the blood-brain barrier ${ }^{12}$ which could have a profound impact on transport, tissue environment, and synaptic activity. The disruption of lactate transport via downregulation of MCT4 has been shown to affect memory formation and synaptic transmission in the hippocampus ${ }^{31}$ and lactate transport is dysregulated in the APP/PS1 mouse model of $\mathrm{AD}^{6}$. 
265 Collectively, these data support the therapeutic potential of SEMA4D antibody blockade to regulate 266 astrocytic function, preventing neuronal toxicity and dysfunction in neurodegenerative diseases 267 including HD and AD. These mechanistic studies may help to inform selection of physiologically 268 relevant biomarkers, which will be important for clinical evaluation and development. As might be 269 expected from expression of glucose transporter in astrocyte projections that fully cover brain capillaries, it was recently demonstrated that astrocytes contribute significantly to the FDG-PET signal 271 in the brain ${ }^{19}$. Decline in FDG-PET is a regular feature of multiple slowly progressive 272 neurodegenerative diseases. Several studies have demonstrated that decline of FDG-PET signal in AD 273 correlates with cognitive decline ${ }^{47,48}$. The clinical benefit of pepinemab treatment and potential 274 correlation to glucose metabolism and neuroprotection, measured using biomarkers FDG-PET and 275 volumetric MRI respectively, was evaluated in a Phase 2 clinical study of a humanized SEMA4D 276 blocking antibody, pepinemab, in subjects known to express the dominant CAG repeat HD mutation 277 (SIGNAL, NCT02481674). This fully randomized 265 patient study has recently concluded, and a 278 detailed report of safety and clinical efficacy is in preparation and will be published elsewhere. Another 279 Phase 1 clinical study is planned in early AD (SIGNAL-AD, NCT04381468) and will investigate safety, 280 as well as the effects of pepinemab on imaging biomarkers. Clearly, it will be important to determine 281 whether biomarkers of astrocytic function and neuronal protection will correlate with positive clinical 282 outcomes.

283 Studies of neurodegenerative diseases have long focused on neurotoxic proteins, however non-neuronal 284 glial cells are increasingly recognized for their important roles in disease onset and escalation ${ }^{1}$.

285 Strategies that target these pathogenic processes have the potential to be broadly applicable to multiple 286 devastating diseases. 
METHODS:

293 Immunohistochemistry. Mouse brain sections: Whole mouse brains from heterozygote (HET) Q175 294 knock-in (KI) (CHDI-81003003) Huntington's mice and wild-type (WT) littermate controls; collected at $295 \sim$ 3, 6, 9 months of age were obtained from CRL Discovery Services. Three consecutive whole mouse 296 brain coronal sections $(5 \mu \mathrm{m})$ from 3 subjects per age group (cases/controls) were used for 297 immunohistochemistry. Immunofluorescence staining of tissue sections were performed for mouse 298 semaphorin 4D (PA5-47711, Invitrogen), NeuN (ab177487, Abcam), GFAP (ab7260, Abcam), 299 somatostatin (H-106, Santa Cruz), Neuropeptide-Y (NPY; NBP-19808, Novus), sypatophysin (101004, 300 SySy), VGLUT-1 (TA317309, Orogene), in accordance to manufacturer recommended concentrations 301 combining host dependent secondary alexa fluor antibodies. Human brain sections: Human HD brain 302 specimens were obtained from National Institute of Health, Neurobiobank (NBB) Brain and Tissue 303 Repositories (protocol number -1105-HDPilotSep2018) through participating brain banks of The Mount 304 Sinai/JJ Peters VA Medical Center NIH Brain and Tissue Repository, Icahn School of Medicine at 305 Mount Sinai; Harvard Brain Tissue Resource Center, Harvard Medical School; University of Miami 306 Brain Endowment Bank, Miller School of Medicine, University of Miami and Brain and Tissue Bank, 307 School of Medicine, University of Maryland. Samples of human frontal cortex regions were obtained 308 from inferior frontal gyrus including Brodmann area -BA 44-45 and parietal lobe regions from 309 somatosensory cortex, including Brodmann area- BA 1,2,3 and part of frontal cortex BA4. Age (years) 310 and gender characteristics ( $\mathrm{F}=\mathrm{female}, \mathrm{M}=\mathrm{male})$ of samples used in this study are as follows: Normal 311 controls: $63(\mathrm{M}), 56(\mathrm{~F}), 54(\mathrm{M})$; HD Stage 0: $81(\mathrm{~F}), 49(\mathrm{M}), 52(\mathrm{~F})$; HD Stage 1: $74(\mathrm{M}), 69(\mathrm{M}), 57$

312 (M); HD Stage 2: $63(\mathrm{~F}), 65(\mathrm{M}), 55(\mathrm{~F})$. FFPE blocks containing roughly $25 \mathrm{mmX} 15 \mathrm{mmX} 5 \mathrm{~mm}$ size of 14 
brain were processed for three-four consecutive $16 \mu \mathrm{m}$ thin sections for immunohistochemistry. Human

314 AD brain specimens were obtained from BioChain Institute, Inc. Newark, CA. The tissue included areas

315 from frontal, temporal, parietal, occipital lobes and corpus callosum; from diencephalic region, the 316 thalamus and from metencephalonic structure, the pons and cerebellum. Age (years) and gender 317 characteristics $(\mathrm{F}=$ female, $\mathrm{M}=$ male) of samples used in this study are as follows: Normal controls: 89 318 (M), $68(\mathrm{M}), 60(\mathrm{M}), 50(\mathrm{~F}), 54(\mathrm{M}), 54(\mathrm{~F}) ;$ AD subjects: $73(\mathrm{M}), 73(\mathrm{M}), 87(\mathrm{M}), 88(\mathrm{M}), 85(\mathrm{~F})$. Three 319 consecutive $5 \mu \mathrm{m}$ thin FFPE blocks sections containing roughly $8 \mathrm{mmX} 10 \mathrm{~mm}$ size of brain, were 320 processed for immunohistochemistry. Immunofluorescence staining of tissue sections were performed 321 for human Semaphorin 4D (ab134128, Abcam), Glutamine Synthetase (MA5-27749, Invitrogen), $\mathrm{HuC} / \mathrm{HuD}$ (A-21271, Invitrogen) and GFAP (ab7260, Abcam) in accordance to manufacturer recommended concentrations combining host dependent secondary alexa fluor antibodies. Imaging of and cell numbers, whole stained tissue in each slide, three consecutive slices per subject were analyzed with Fiji/Image J software (National Institutes of Health, Bethesda, MD, http://imagej.nih.gov/ij/).

Fractal dimensions: For astrocytes fractal dimensions analysis, $5 \mu \mathrm{m}$-thick brain coronal sections were imaged on AxioObserver7 Automated Inverted Microscope with Plan-Apochromat 20x/0.8; 40x/0.95 objectives and Axiocam 702 Monochrome Camera. Images were taken in different optical planes in order to resolve the finest details possible at this resolution. Based on the extended depth of field algorithm, Zen 3.0 (Carl Zeiss Microscopy, GmbH, 2019) generated a final image which recapitulated all the details of the cell. Cells without visible nuclei were rejected in this study. More than 100 cells per cortex region for each section for each age group (cases/controls) were used for fractal dimension analysis. Fractal analysis was performed as described previously ${ }^{49,50}$ using Fiji/Image J software (National Institutes of Health, Bethesda, MD, http://imagej.nih.gov/ij/) along with the FracLac plug-in (A. Karperien - Charles Sturt 
University, Australia). Fractal dimensions (FDs) were calculated by the box-counting algorithm as the slope of the regression line for the log-log plot of the scanning box size and the count from a box counting scan.

In-vitro rat astrocyte studies: Primary Rat Cortical Astrocytes (Invitrogen, catalog\# N7745-100) were expanded in DMEM supplemented with $15 \%$ FBS for one week and then seeded into 48well plates at 5000 cells/well. The following day five replicate wells received either fresh media or $25 \mathrm{ug} / \mathrm{mL}$ marmoset CD100. After 22 hours of incubation, plates were washed two times with DPBS and then fixed with 4\% PFA. Fixed astrocytes were blocked with 2.5\% Normal Donkey Serum. Astrocytes were stained for filamentous actin (1:100 Phalloidin 568, Invitrogen, catalog \# A12380) and nuclei (1ug/mL DAPI, Invitrogen catalog\# D3571) in PBS + 0.05\% Tween20 for half an hour. For each of five wells, 25 fields of view were acquired using an automated stage by Prior on an inverted Olympus IX50 microscope at 20x. Phalloidin area and cell count were quantified using media cybernetics Image Pro Premier software.

In-vitro human astrocyte studies: Normal human astrocytes (CC-2565, Lonza) were cultured in astrocyte basal growth media (Lonza CC-3186 ABM ${ }^{\mathrm{TM}}$ ) with astrocyte growth medium supplements (AGM; Lonza Inc., Anaheim, CA, USA). Tissue origin of normal human astrocytes is cerebral cortex (grey matter), and the cells are considered type I astrocytes. Astrocytes were seeded on \#1.5; 0.16 to $0.19 \mathrm{~mm}$ cover glasses in 24 well plates at a seeding density of 5,000 cells $/ \mathrm{cm}^{2}$. At around $75 \%$ visual confluency, the cells were treated for 24 or 48 hours with Control protein rEGFR-his $(5 \mu \mathrm{g} / \mathrm{ml})$ or rSEMA4D-his $(5 \mu \mathrm{g} / \mathrm{ml}, 25 \mu \mathrm{g} / \mathrm{ml})$, combination treatments viz SEMA4D $(5 \mu \mathrm{g} / \mathrm{ml})$ and pepinemab "VX15" or control antibody "HuIgG4" (25 $\mu \mathrm{g} / \mathrm{ml})$. Immunofluorescence staining of fixed cells (acetone:methanol::1:1) were performed for semaphorin 4D (ab134128, Abcam), EAAT-2 (711020, Invitrogen), Glut1 (ab15309, Abcam), and MCT4 (ab244385, Abcam) in accordance to manufacturer recommendation. Imaging of stained cover glasses were performed on AxioObserver7 Automated 
361 Inverted Microscope System with EC Plan-Neofluar 10x/0.30 and Objective Plan-Apochromat 40x/0.95.

362 For immunofluorescence intensity, whole stained cover glasses in triplicate per treatment conditions

363 were analyzed with Fiji/Image J software (National Institutes of Health, Bethesda, MD,

364 http://imagej.nih.gov/ij/). The morphometric characterization of astrocytes in in-vitro conditions were

365 done on 40X images acquired by a Zeiss AxioObserver imaging Apotome system with the aid of

366 AutoNeuriteJ, an imageJ/Fiji plugin that can measure and classifies complex cell structures including

367 length and number of cell processes ${ }^{51}$.

368 Glucose uptake assay: Normal human primary astrocytes (CC-2565, Lonza) were cultured in astrocyte 369 basal growth media as described in previous section. At around 75\% confluency, the cells were washed 370 twice with glucose-free HBS before treatment with varying concentrations/combinations of control 371 protein rEGFR, rSEMA4D, anti-SEMA4D antibody/VX15 and isotype control human IgG4 antibody. 372 All the treatment conditions were prepared in DMEM (Gibco A1443001), containing no glucose or 373 glutamine. The medium was further supplemented with $10 \%$ fetal bovine dialyzed serum (Gibco 374 A3382001), and glucose (10mM). After 24-48 hrs of treatment period, the treatment medium was 375 discarded and cells were washed twice with glucose-free HBS. Cells were further treated for 5 minutes 376 with $0.6 \mathrm{~N} \mathrm{HCl}$ to rapidly stop cell metabolism and lysis followed by neutralization with $1 \mathrm{M}$ Tris base $377(v / v)$ for 1 minute. Glucose detection was performed using Glucose-Glo ${ }^{\mathrm{TM}}$ Assay (J6022, Promega) 378 according to the manufacturer's recommendations. Buffer only control was used as negative control to 379 measure background signal, data has been presented as normalize fluorescence units (AU) after 380 calculating signal-to-background ratios.

381 CVN mouse model: Homozygous APPSwDI/NOS2-/- bigenic mice harbor the APPSwDI transgene 382 (encoding the Swedish K670N/M671L vasculotropic Dutch/Iowa E693Q/D694N mutant) and a targeted 383 "null" mutation of the nitric oxide synthase 2 (Nos2, or inducible NOS, iNOS) locus (Charles River 384 Laboratories). Male and female wild type and CVN mice were treated in a gender-balanced and blinded 
manner with anti-SEMA4D (MAb67) antibody or mouse IgG1 isotype control (MAb 2B8) (30 mg/kg,

386 IV, weekly x 12) at 26 weeks of age. Brains were harvested at 41 weeks of age, perfused with non387 heparinized saline and post-fixed by immersion in $4 \%$ PFA for IHC analysis. Analysis focused on 388 hippocampal regions including CA1, dentate gyrus and subiculum - NPY, V-GLUT and synaptophysin 389 were quantified in dentate gyrus region and somatostatin was quantified in subiculum. Percentage of 390 somatostatin-positive signal was quantified within the subiculum of all animals and normalized to total 391 subiculum area scanned and percentage of NPY-positive signal was quantified within the dentate gyrus 392 of all animals and normalized to total dentate gyrus area scanned. Live animal phase was conducted 393 according to the following guidelines: Act on Use of Animals for Experimental Purposes (62/2006), 394 Finland; Ordinance number 36/EEO/2006 on experimental animals, Ministry of Agriculture and 395 Forestry, Finland; Directive 2010/63/EU of the European Parliament and of the Council of 22 396 September 2010 on the protection of animals used for scientific purpose. All animal experiments are 397 approved by the State Provincial Office of Southern Finland.

398 Statistical analysis: Analysis of in vitro data was performed by IBM SPSS software (version 1.0.0.1327). 399 Group differences among treatment conditions were assessed through factorial MANOVA and time point 400 variations using ANCOVA. Linear relationship between the dependent variables were assessed by 401 scatterplot and multivariate outliers were screened with Mahalanobis distance (p > .001). Statistical 402 403 analysis of IHC data was performed with IBM SPSS software (version 1.0.0.1327); one-way ANOVA was used to compare multiple (>2) groups with one independent variable followed by Tukey's multiple 404 comparison. Data outlier's and normal data distributions were assessed through boxplot and Shapiro-Wilk 405 test. Homogeneity of variances between groups within each data set was determined with Levene's test. 406 Statistical significance was determined with one-way ANOVA for p-values < 0.05 with Tukey's Post-hoc 407 test. Dataset with violations in homogeneity of variances, statistical significance was determined with 
408 Welch one-way ANOVA for p-values < 0.05 with Games-Howell post hoc analysis. All error bars in 409 figures represent the standard error of the mean (SEM). $* \mathrm{p}<0.05 ; * * \mathrm{p}<0.01 ; * * * \mathrm{p}<0.001 ; * * * * \mathrm{p}<0.0001$ 


\section{REFERENCES}

1 Chen, W. W., Zhang, X. \& Huang, W. J. Role of neuroinflammation in neurodegenerative diseases (Review). Mol Med Rep 13, 3391-3396, doi:10.3892/mmr.2016.4948 (2016).

2 Pekny, M. et al. Astrocytes: a central element in neurological diseases. Acta Neuropathol 131, 323-345, doi:10.1007/s00401-015-1513-1 (2016).

3 Benraiss, A. et al. Human glia can both induce and rescue aspects of disease phenotype in Huntington disease. Nat Commun 7, 11758, doi:10.1038/ncomms11758 (2016).

4 Zamanian, J. L. et al. Genomic analysis of reactive astrogliosis. J Neurosci 32, 6391-6410, doi:10.1523/JNEUROSCI.6221-11.2012 (2012).

5 Liu, W. et al. Activation of brain glucose metabolism ameliorating cognitive impairment in APP/PS1 transgenic mice by electroacupuncture. Free Radic Biol Med 112, 174-190, doi:10.1016/j.freeradbiomed.2017.07.024 (2017).

6 Zhang, M. et al. Lactate Deficit in an Alzheimer Disease Mouse Model: The Relationship With Neuronal Damage. J Neuropathol Exp Neurol 77, 1163-1176, doi:10.1093/jnen/nly102 (2018).

7 Ortinski, P. I. et al. Selective induction of astrocytic gliosis generates deficits in neuronal inhibition. Nat Neurosci 13, 584-591, doi:10.1038/nn.2535 (2010).

8 Pekny, M. \& Pekna, M. Astrocyte reactivity and reactive astrogliosis: costs and benefits. Physiol Rev 94, 1077-1098, doi:10.1152/physrev.00041.2013 (2014).

9 Basile, J. R., Gavard, J. \& Gutkind, J. S. Plexin-B1 utilizes RHOA and ROK to promote the integrindependent activation of AKT and ERK, and endothelial cell motility. J. Biol. Chem 282, 34888-34895 (2007).

10 Liang, X., Draghi, N. A. \& Resh, M. D. Signaling from integrins to Fyn to Rho family GTPases regulates morphologic differentiation of oligodendrocytes. J Neurosci 24, 7140-7149, doi:10.1523/JNEUROSCI.5319-03.2004 [doi];24/32/7140 [pii] (2004).

11 Tamagnone, L. et al. Plexins are a large family of receptors for transmembrane, secreted, and GPIanchored semaphorins in vertebrates. Cell 99, 71-80 (1999).

12 Smith, E. S. et al. SEMA4D compromises blood-brain barrier, activates microglia, and inhibits remyelination in neurodegenerative disease. Neurobiol. Dis 73, 254-268 (2014).

13 Okuno, T. et al. Roles of SEMA4D-plexin-B1 interactions in the central nervous system for pathogenesis of experimental autoimmune encephalomyelitis. J. Immunol 184, 1499-1506, doi:jimmunol.0903302 [pii];10.4049/jimmunol.0903302 [doi] (2010).

14 Giraudon, P. et al. Semaphorin CD100 from activated T lymphocytes induces process extension collapse in oligodendrocytes and death of immature neural cells. J. Immunol 172, 1246-1255 (2004).

15 Giraudon, P., Vincent, P. \& Vuaillat, C. T-cells in neuronal injury and repair: semaphorins and related Tcell signals. Neuromolecular. Med 7, 207-216 (2005).

16 Magistretti, P. J. \& Allaman, I. A cellular perspective on brain energy metabolism and functional imaging. Neuron 86, 883-901, doi:10.1016/j.neuron.2015.03.035 (2015).

17 Loaiza, A., Porras, O. H. \& Barros, L. F. Glutamate triggers rapid glucose transport stimulation in astrocytes as evidenced by real-time confocal microscopy. J Neurosci 23, 7337-7342 (2003).

18 Robinson, M. B. \& Jackson, J. G. Astroglial glutamate transporters coordinate excitatory signaling and brain energetics. Neurochem Int 98, 56-71, doi:10.1016/j.neuint.2016.03.014 (2016).

19 Zimmer, E. R. et al. [(18)F]FDG PET signal is driven by astroglial glutamate transport. Nat Neurosci 20, 393-395, doi:10.1038/nn.4492 (2017). 
Southwell, A. L. et al. Anti-semaphorin 4D immunotherapy ameliorates neuropathology and some cognitive impairment in the YAC128 mouse model of Huntington disease. Neurobiol. Dis 76, 46-56, doi:S0969-9961(15)00014-5 [pii];10.1016/j.nbd.2015.01.002 [doi] (2015).

21 Shin, J. Y. et al. Expression of mutant huntingtin in glial cells contributes to neuronal excitotoxicity. J Cell Biol 171, 1001-1012, doi:10.1083/jcb.200508072 (2005).

22 Tong, X. et al. Astrocyte Kir4.1 ion channel deficits contribute to neuronal dysfunction in Huntington's disease model mice. Nat. Neurosci 17, 694-703, doi:nn.3691 [pii];10.1038/nn.3691 [doi] (2014). Osipovitch, M. et al. Human ESC-Derived Chimeric Mouse Models of Huntington's Disease Reveal CellIntrinsic Defects in Glial Progenitor Cell Differentiation. Cell Stem Cell 24, 107-122 e107, doi:10.1016/j.stem.2018.11.010 (2019).

24 Khakh, B. S. et al. Unravelling and Exploiting Astrocyte Dysfunction in Huntington's Disease. Trends Neurosci 40, 422-437, doi:10.1016/j.tins.2017.05.002 (2017).

25 McColgan, P. \& Tabrizi, S. J. Huntington's disease: a clinical review. Eur J Neurol 25, 24-34, doi:10.1111/ene.13413 (2018).

26 Wilhelmsson, U. et al. Redefining the concept of reactive astrocytes as cells that remain within their unique domains upon reaction to injury. Proc Natl Acad Sci U S A 103, 17513-17518, doi:10.1073/pnas.0602841103 (2006). Bribian, A., Perez-Cerda, F., Matute, C. \& Lopez-Mascaraque, L. Clonal Glial Response in a Multiple Sclerosis Mouse Model. Front Cell Neurosci 12, 375, doi:10.3389/fncel.2018.00375 (2018). Coulter, D. A. \& Eid, T. Astrocytic regulation of glutamate homeostasis in epilepsy. Glia 60, 1215-1226, doi:10.1002/glia.22341 (2012).

Pirici, D. et al. Fractal analysis of astrocytes in stroke and dementia. Rom J Morphol Embryol 50, 381-390 (2009).

Soltys, Z., Ziaja, M., Pawlinski, R., Setkowicz, Z. \& Janeczko, K. Morphology of reactive microglia in the injured cerebral cortex. Fractal analysis and complementary quantitative methods. J Neurosci Res 63, 9097, doi:10.1002/1097-4547(20010101)63:1<90::AID-JNR11>3.0.CO;2-9 (2001).

Netzahualcoyotzi, C. \& Pellerin, L. Neuronal and astroglial monocarboxylate transporters play key but distinct roles in hippocampus-dependent learning and memory formation. Prog Neurobiol, 101888, doi:10.1016/j.pneurobio.2020.101888 (2020).

32 Colton, C. A. et al. The effects of NOS2 gene deletion on mice expressing mutated human AbetaPP. J Alzheimers Dis 15, 571-587, doi:10.3233/jad-2008-15405 (2008).

33 Colton, C. A. et al. mNos2 deletion and human NOS2 replacement in Alzheimer disease models. J Neuropathol Exp Neurol 73, 752-769, doi:10.1097/NEN.0000000000000094 (2014).

34 Wilcock, D. M. \& Colton, C. A. Anti-amyloid-beta immunotherapy in Alzheimer's disease: relevance of transgenic mouse studies to clinical trials. J Alzheimers Dis 15, 555-569, doi:10.3233/jad-2008-15404 (2008). Liddelow, S. A. et al. Neurotoxic reactive astrocytes are induced by activated microglia. Nature 541, 481487, doi:10.1038/nature21029 (2017).

36 Yamanaka, K. et al. Astrocytes as determinants of disease progression in inherited amyotrophic lateral sclerosis. Nat Neurosci 11, 251-253, doi:10.1038/nn2047 (2008).

37 Diaz-Castro, B., Gangwani, M. R., Yu, X., Coppola, G. \& Khakh, B. S. Astrocyte molecular signatures in Huntington's disease. Sci Transl Med 11, doi:10.1126/scitranslmed.aaw8546 (2019).

38 Ament, S. A. et al. Transcriptional regulatory networks underlying gene expression changes in Huntington's disease. Mol Syst Biol 14, e7435, doi:10.15252/msb.20167435 (2018).

39 Habib, N. et al. Disease-associated astrocytes in Alzheimer's disease and aging. Nat Neurosci 23, 701706, doi:10.1038/s41593-020-0624-8 (2020).

40 Barbar, L. et al. CD49f Is a Novel Marker of Functional and Reactive Human iPSC-Derived Astrocytes. Neuron 107, 436-453 e412, doi:10.1016/j.neuron.2020.05.014 (2020). 
41 Johnson, E. C. B. et al. Large-scale proteomic analysis of Alzheimer's disease brain and cerebrospinal fluid reveals early changes in energy metabolism associated with microglia and astrocyte activation. Nat Med 26, 769-780, doi:10.1038/s41591-020-0815-6 (2020).

42 Mason, S. Lactate Shuttles in Neuroenergetics-Homeostasis, Allostasis and Beyond. Front Neurosci 11, 43, doi:10.3389/fnins.2017.00043 (2017).

43 Reichmann, F. \& Holzer, P. Neuropeptide Y: A stressful review. Neuropeptides 55, 99-109, doi:10.1016/j.npep.2015.09.008 (2016).

44 Borbely, E., Scheich, B. \& Helyes, Z. Neuropeptides in learning and memory. Neuropeptides 47, 439-450, doi:10.1016/j.npep.2013.10.012 (2013).

45 Kowall, N. W. \& Beal, M. F. Cortical somatostatin, neuropeptide Y, and NADPH diaphorase neurons: normal anatomy and alterations in Alzheimer's disease. Ann Neurol 23, 105-114, doi:10.1002/ana.410230202 (1988).

46 Wilcock, D. M. et al. Progression of amyloid pathology to Alzheimer's disease pathology in an amyloid precursor protein transgenic mouse model by removal of nitric oxide synthase 2. J. Neurosci 28, 15371545, doi:28/7/1537 [pii];10.1523/JNEUROSCI.5066-07.2008 [doi] (2008).

47 Landau, S. M. et al. Associations between cognitive, functional, and FDG-PET measures of decline in AD and MCl. Neurobiol Aging 32, 1207-1218, doi:10.1016/j.neurobiolaging.2009.07.002 (2011).

48 Khosravi, M. et al. 18F-FDG Is a Superior Indicator of Cognitive Performance Compared to 18FFlorbetapir in Alzheimer's Disease and Mild Cognitive Impairment Evaluation: A Global Quantitative Analysis. J Alzheimers Dis 70, 1197-1207, doi:10.3233/JAD-190220 (2019).

49 Karperien, A., Ahammer, H. \& Jelinek, H. F. Quantitating the subtleties of microglial morphology with fractal analysis. Front Cell Neurosci 7, 3, doi:10.3389/fncel.2013.00003 (2013).

50 Young, K. \& Morrison, H. Quantifying Microglia Morphology from Photomicrographs of Immunohistochemistry Prepared Tissue Using ImageJ. J Vis Exp, doi:10.3791/57648 (2018).

51 Boulan, B. et al. AutoNeuriteJ: An ImageJ plugin for measurement and classification of neuritic extensions. PLoS One 15, e0234529, doi:10.1371/journal.pone.0234529 (2020). 


\section{ACKNOWLEDGMENTS:}

Funding provided by Vaccinex, Inc., Rochester, New York

\section{AUTHOR CONTRIBUTIONS:}

M. Zauderer, E.E. Evans, V. Mishra, T.L. Fisher were involved in design of the study.

E.E. Evans and V. Mishra performed statistical analysis.

M. Zauderer, E.E. Evans, T.L. Fisher, and V. Mishra interpreted the data.

E.E. Evans, V. Mishra, E.S. Smith, L. Balch, C. Mallow, E. Gersz, and A. Howell designed studies and acquired data.

M. Zauderer, and E.E. Evans wrote the first draft of the manuscript and V. Mishra, T. Fisher, E.S. Smith revised the manuscript for content and clarity.

\section{COMPETING INTEREST STATEMENTS:}

M. Zauderer, E.E. Evans, T.L. Fisher, V. Mishra, L. Balch, C. Mallow, A. Howell, E. Gersz, and E.S. Smith are employees of Vaccinex, Inc. and own stock and/or stock options in the company. 
a
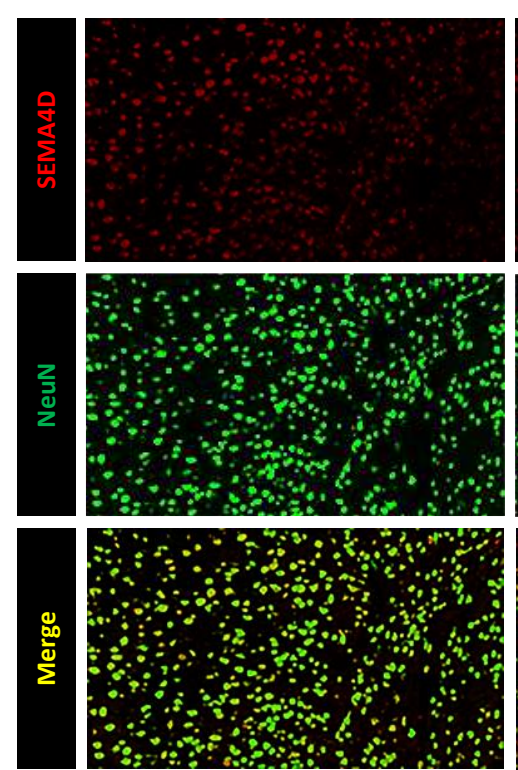

WT-3M
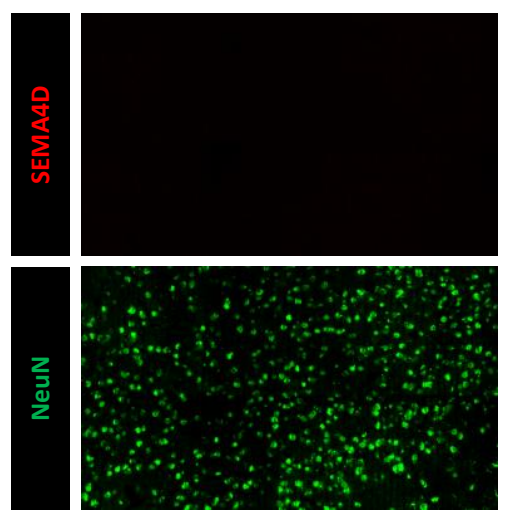

ญั
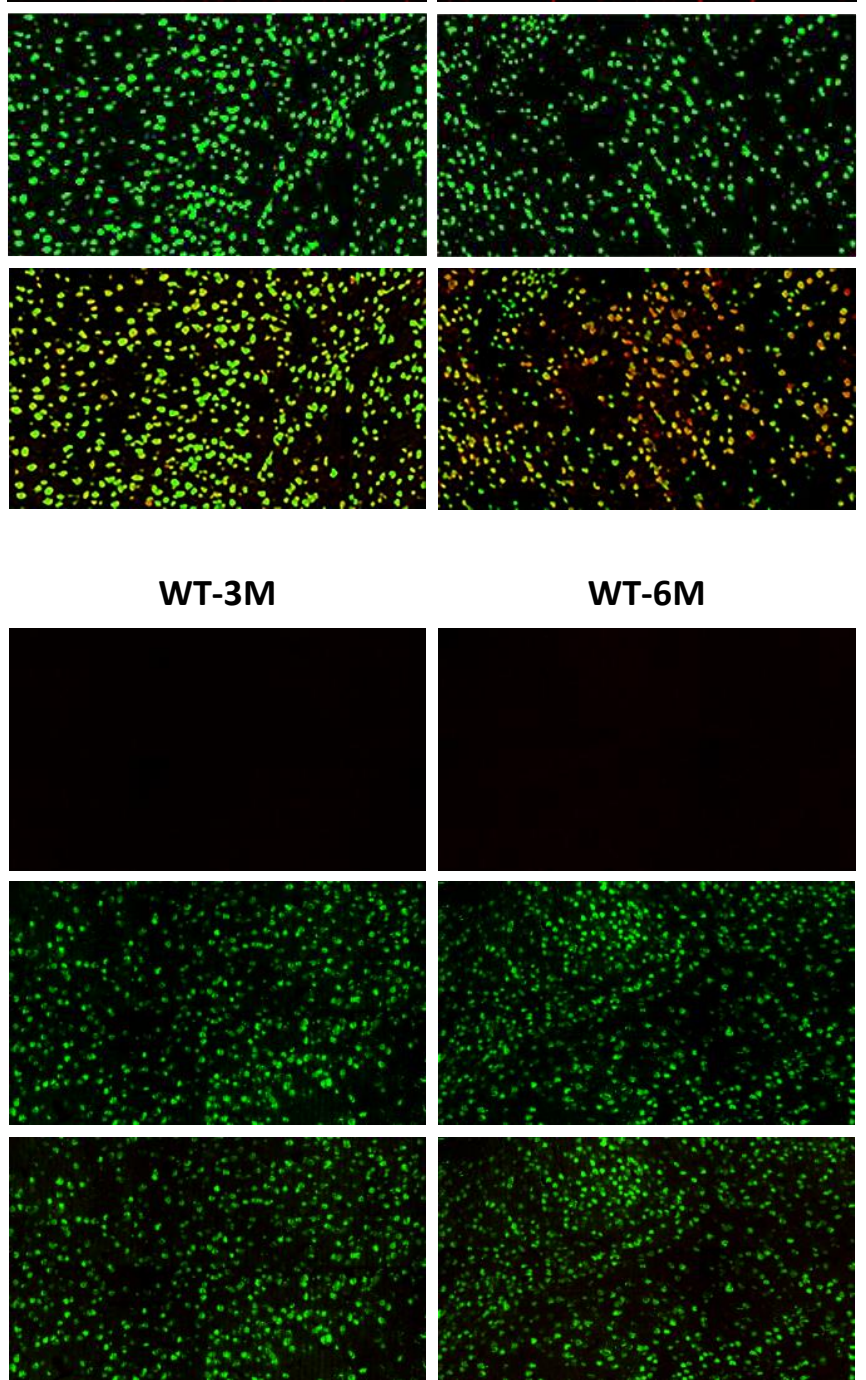

WT-6M
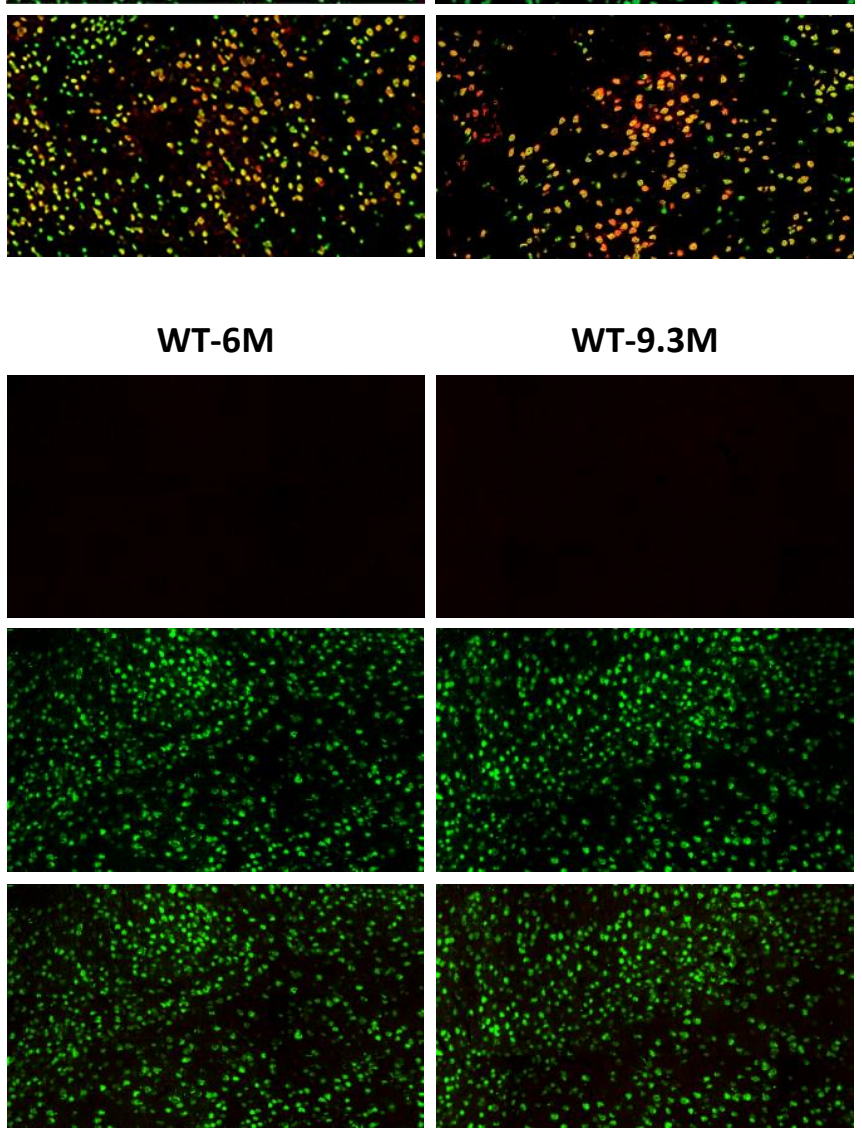

WT-9.3M
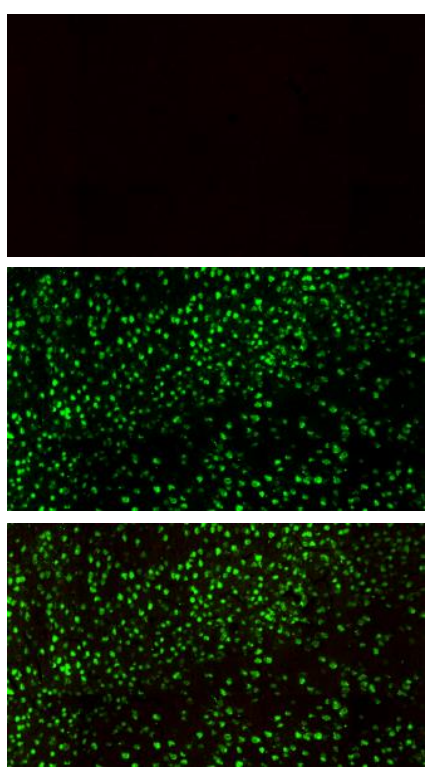

b
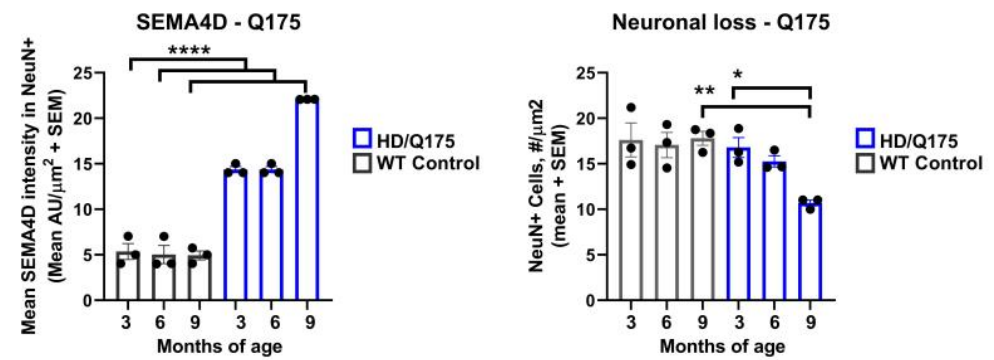

Figure 1: SEMA4D is increasingly upregulated in HD neurons in parallel with neuronal loss. (continued on next page) 

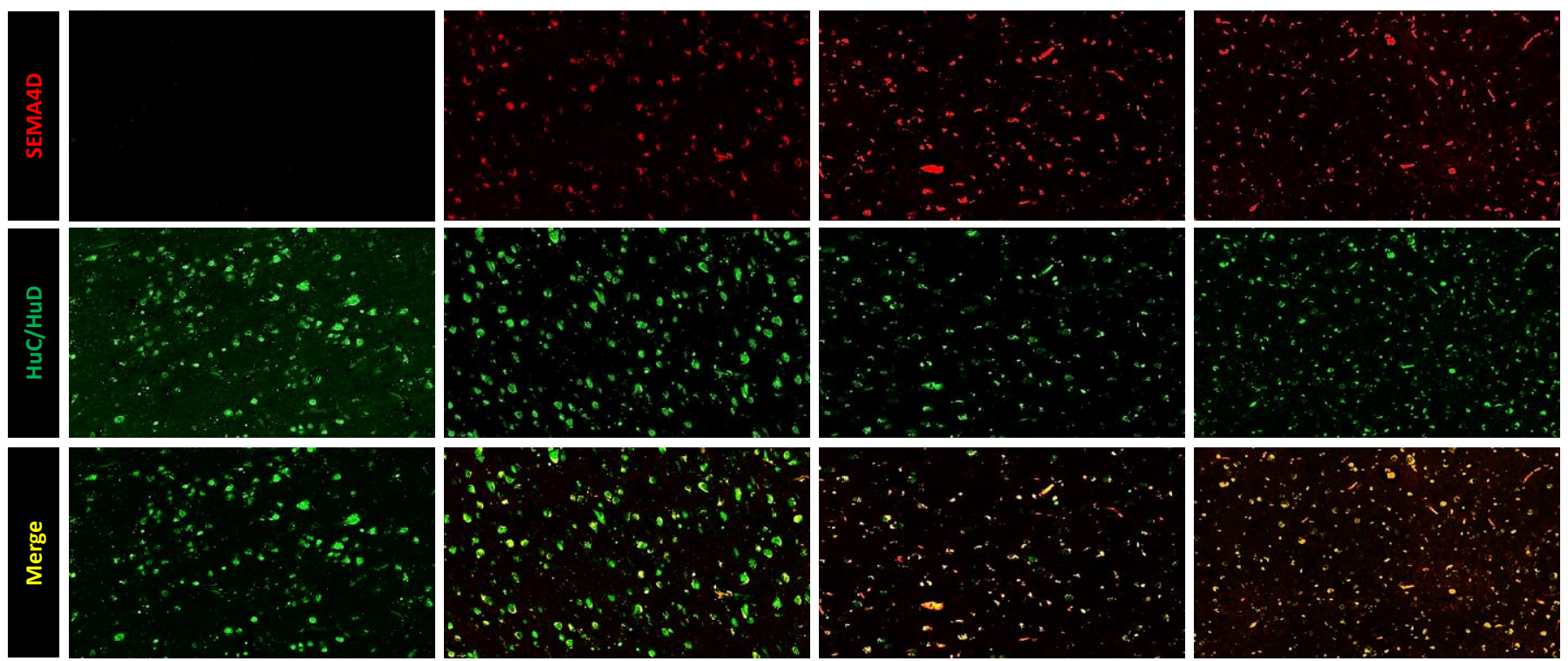

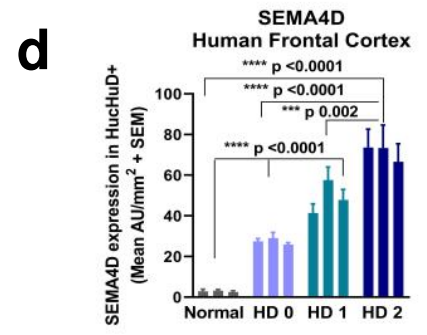

Huc/HuD ${ }^{+}$Neurons Human Frontal Cortex

e

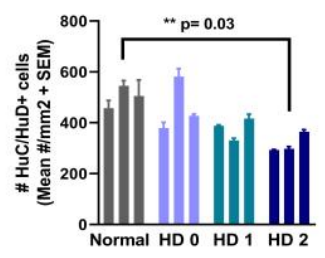

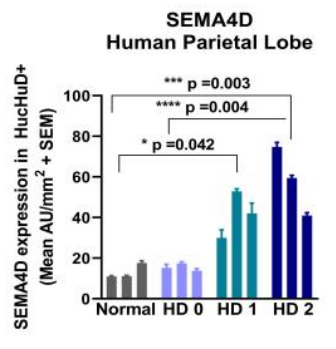

Huc/HuD ${ }^{+}$Neurons Human Parietal Lobe

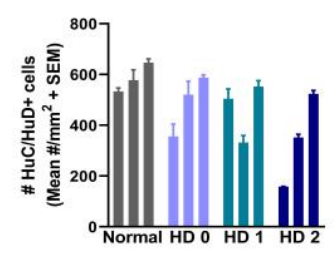

SEMA4D

Human Caudate/Putamen

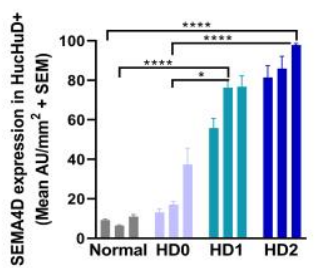

Huc/HuD ${ }^{+}$Neurons

Human Caudate/Putamen

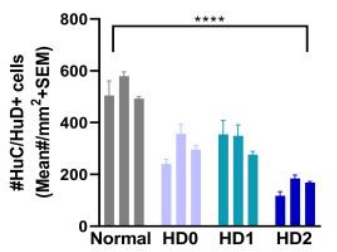

Figure 1: SEMA4D is increasingly upregulated in HD neurons in parallel with neuronal loss. a. Q175 knock-in mouse model of HD exhibits age-dependent upregulation and colocalization of SEMA4D in cortical neurons. b. Quantification of SEMA4D expression and number of neurons in Q175 model. Mean of entire coronal section +SEM (bar) with 3 mice/age group (closed circles). c. SEMA4D is increasingly upregulated with increasing human HD pathological stage in parallel with neuronal loss. Representative images of frontal lobe are shown. d. SEMA4D expression and e. number of neurons were quantified across entire human autopsy sections of frontal cortex (Inferior frontal gyrus, Brodmann area -BA 44-45), parietal lobe (Somatosensory cortex-

Brodmann area- BA 1,2,3 and part of frontal cortex BA4) and striatum (caudate/putamen) regions. Mean of 3-4 consecutive sections +SEM is shown for 3 subjects/condition. Group differences and statistical significance was determined using one-way ANOVA with Tukey post hoc analysis and is indicated by ${ }^{*} p<0.05,{ }^{* *} p<0.01,{ }^{* * *}$ $p<0.001,{ }^{* * * *} p<0.0001$. 

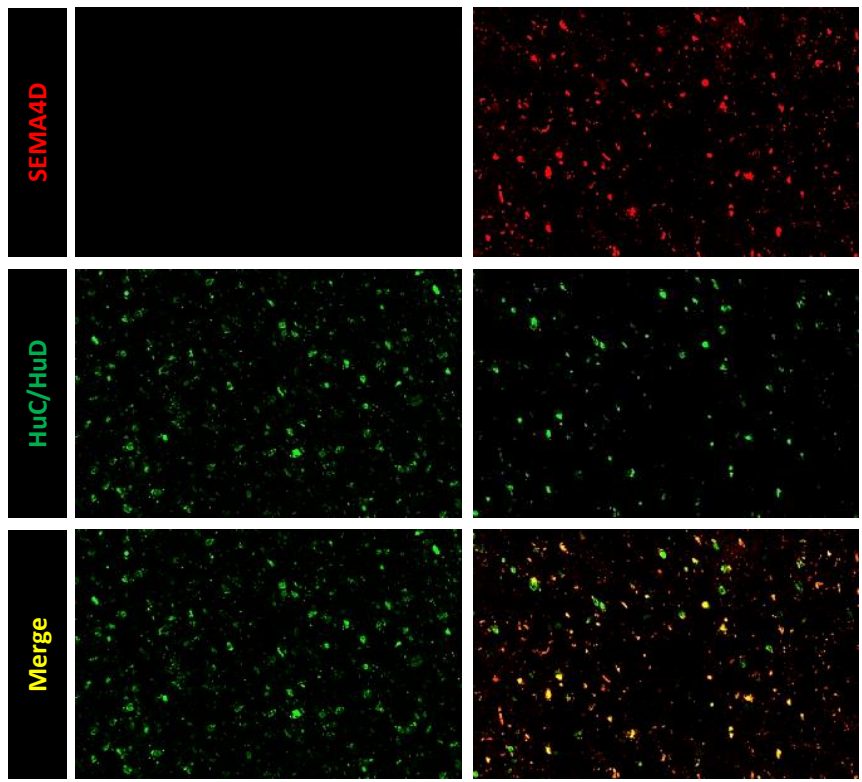

b

Human Thalamus

Human Temporal Lobe

Human Frontal Cortex
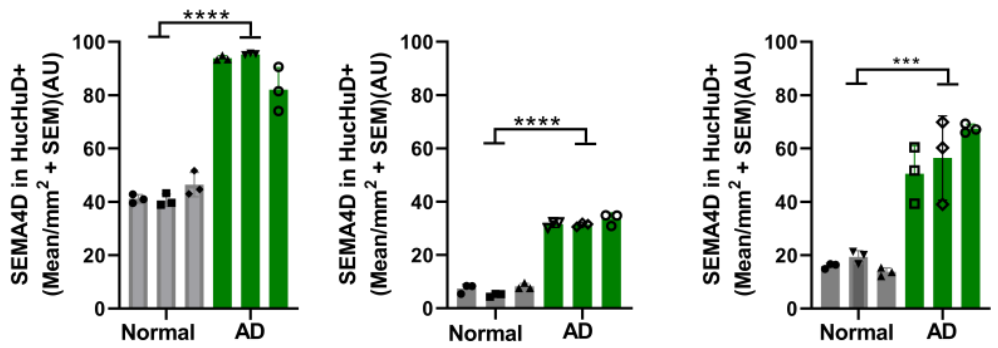

C

Human Thalamus

Human Temporal Lobe

Human Frontal Cortex
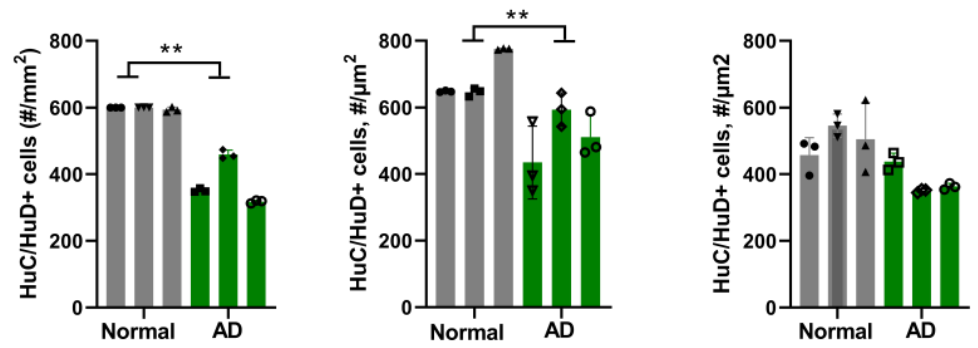

Figure 2: SEMA4D is upregulated in neurons of human AD. a. Representative images of human temporal lobe sections stained for SEMA4D and Huc/HuD (neuronal cell body). b. SEMA4D expression and $\mathbf{c}$. number of neurons were quantified across entire human $A D$ autopsy sections of thalamus, temporal lobe (Brodmann area -BA 38), frontal cortex (Inferior frontal gyrus, Brodmann area -BA 44-45). Imaging of three consecutive slices of brain regions approximately $8 \mathrm{~mm} \times 10 \mathrm{~mm}$ in size was performed. Mean of 3-4 consecutive sections +SEM is shown for 3 subjects/condition. Group differences and statistical significance was determined using one-way ANOVA with Tukey post hoc analysis and is indicated by ${ }^{*} p<0.05,{ }^{* *} p<0.01,{ }^{* * *} p<0.001,{ }^{* * *} p<0.0001$. 
a

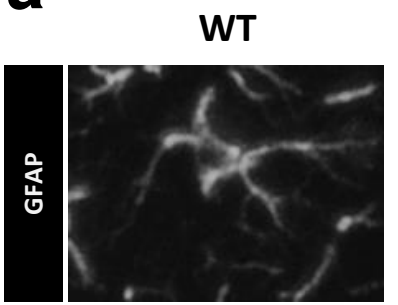

HD Q175 -3M HD Q175-6M

HD Q175 -9.3M
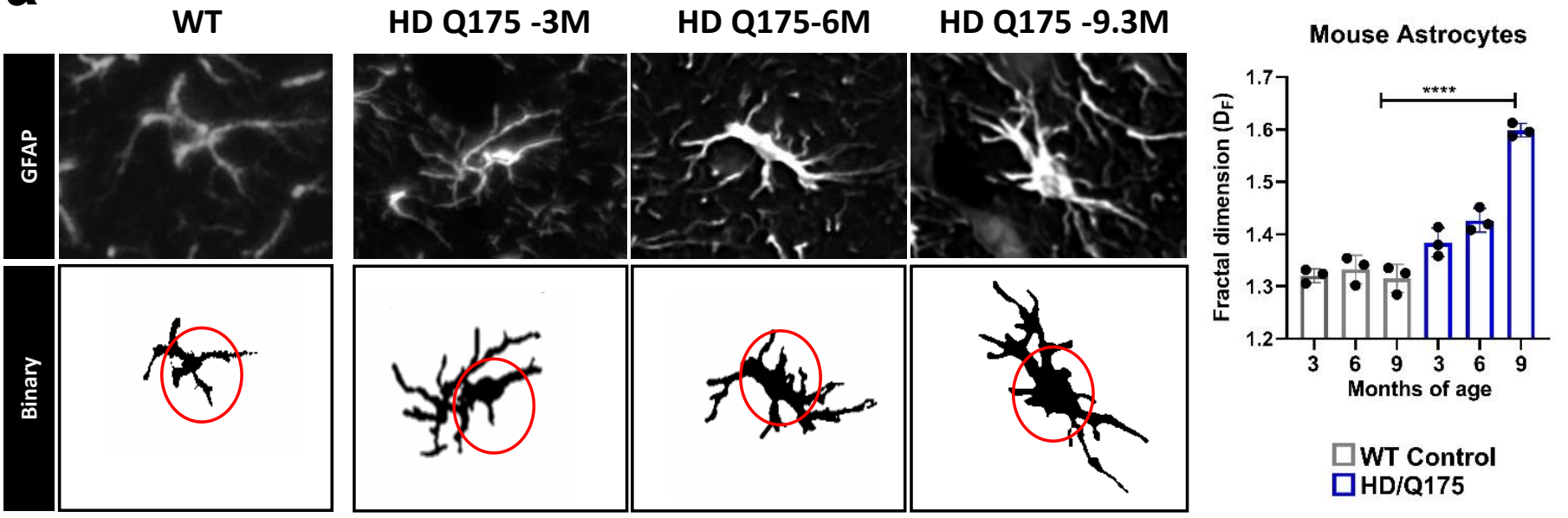

b

Normal Human

HD stage 0

AD
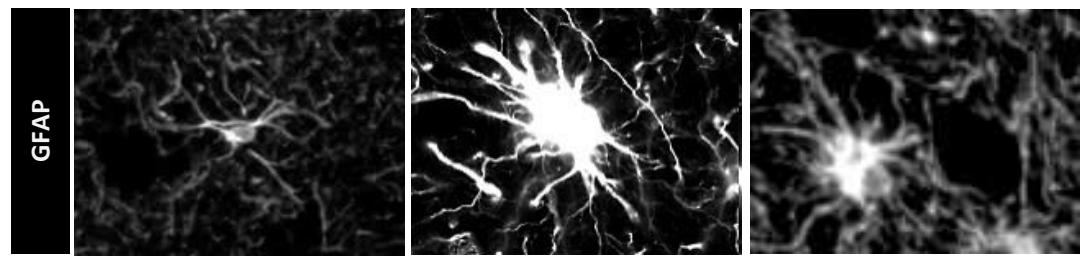

胥
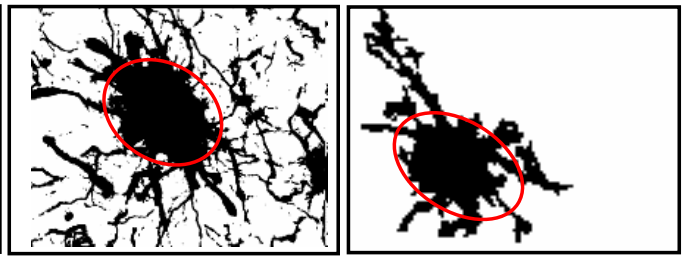

Human Astrocytes

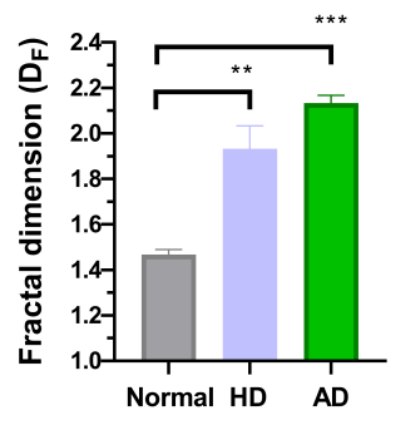

Figure 3: SEMA4D+ cells are in close proximity to astrocytes; morphologic changes indicative of astrogliosis are apparent with disease progression. a. GFAP staining of caudoputamen from analysis of 3 mice/time-point are shown and were obtained from mouse brain coronal sections $(\sim 5 \mu \mathrm{M})$; representative images shown at 20X magnification. Fractal dimension analysis of astrocytes in mouse frontal cortex demonstrate significant changes in brains of Q175 mice at 9.3 months of age. b. Representative images of frontal lobe from non-diseased human, Stage0 HD and AD subjects are shown (20X magnification). Fractal dimension analysis of astrocytes in frontal cortex demonstrate significant changes between non-diseased ("normal") and HD or AD. 

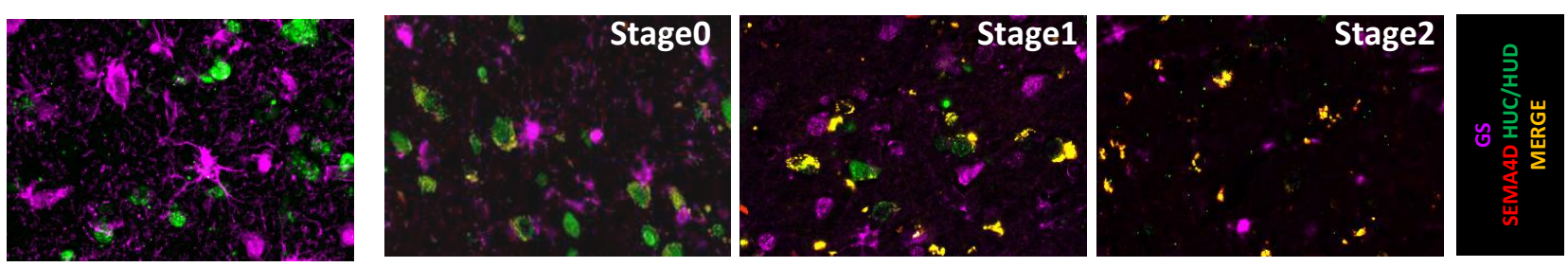

b

Human Frontal Cortex

Human Parietal Lobe

Human Caudoputamen
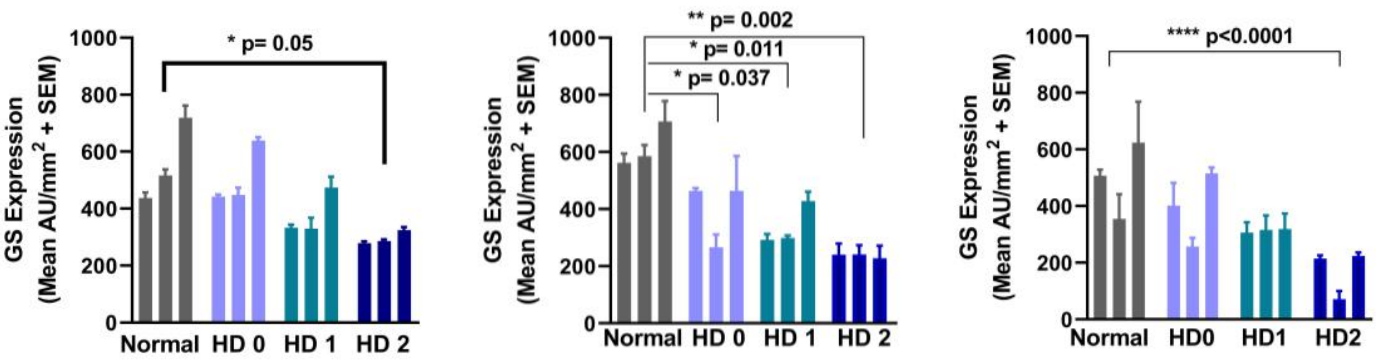

C
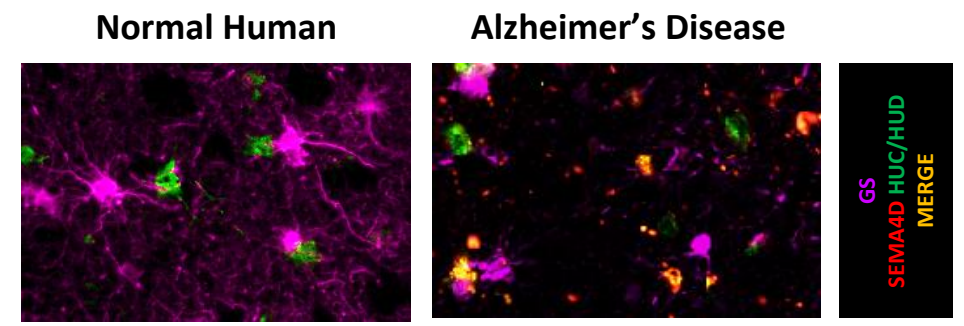

d

Human Thalamus

Human Temporal Lobe

Human Frontal Cortex
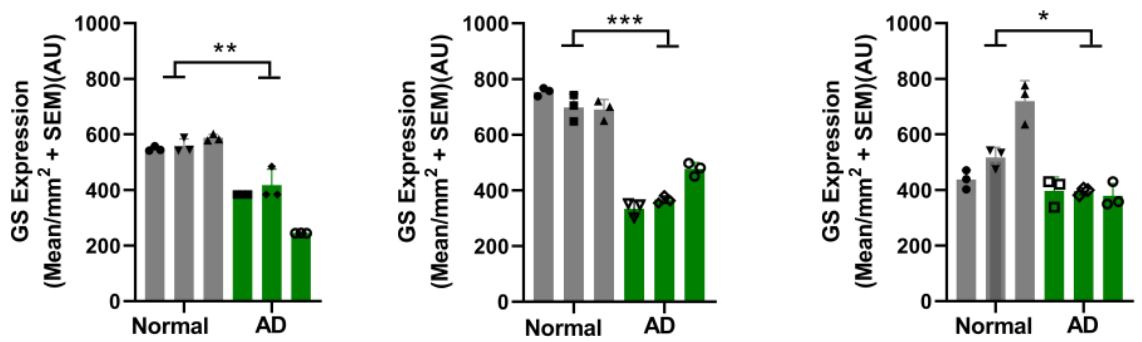

Figure 4: Downregulation of GS in reactive astrocytes is coincident with SEMA4D upregulation in neurons of HD and AD brains. Reactive astrocyte morphology, with reduced GS expression and retracted astrocytic endfeet, along with upregulation of neuronally expressed SEMA4D in HD (a,b) and AD (b,c) a. Human frontal cortex sections were stained for glutamine synthetase (GS, astrocyte cell body and processes), SEMA4D, and Huc/HuD (neuronal cell body). b. Number of GS+ cells were quantified as described in Fig. 1e. c. Human temporal lobe sections were stained for SEMA4D, and Huc/HuD, glutamine synthetase. d. Number of GS+ cells were quantified as described in Fig. 2c. 
a

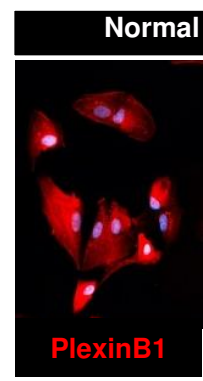

\section{rSEMA4D}

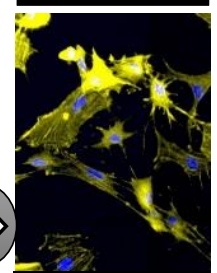

Phalloidin

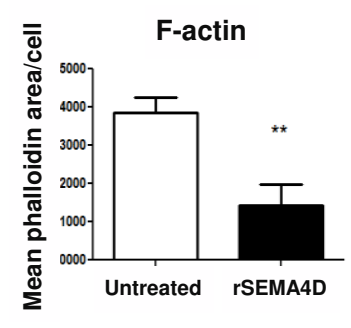

b

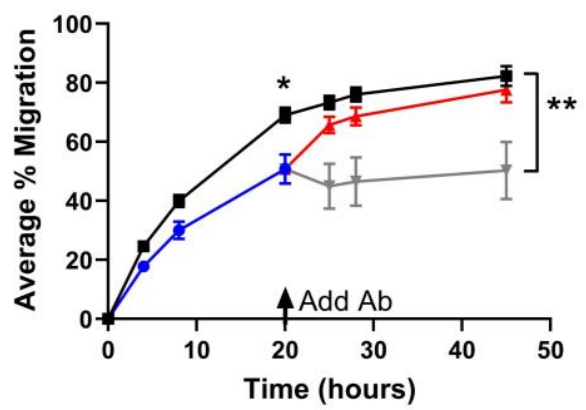

$\rightarrow$ Spontaneous
$\rightarrow$ rSEMA4D $\longrightarrow$ rSEMA4D/CTRL Ig

C

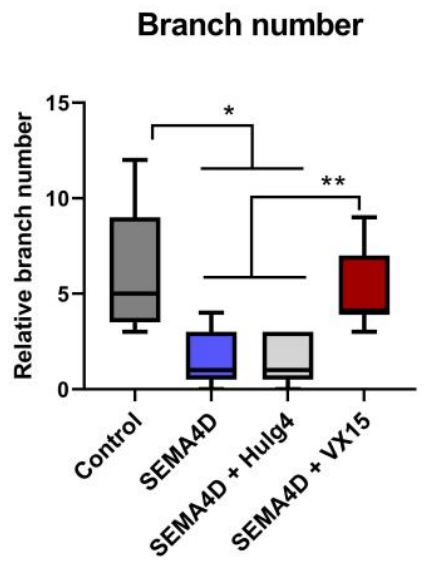

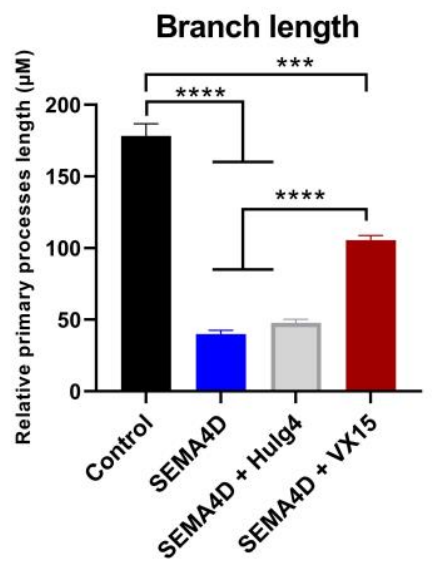

Figure 5: Reactive astrocyte morphologic changes are mediated by SEMA4D signaling through its receptor PlexinB1 and are reversible with antibody blockade of SEMA4D. a. Primary rat astrocyte cultures were stained for expression of receptor PLXNB1 (red), F-actin filaments (phalloidin, yellow) and nuclei (Dapi, blue) before (center) and after (right) one-hour treatment with recombinant SEMA4D. Representative images are shown. Mean phalloidin-positive area/cell in a field of $\sim 300$ cells was quantified using ImagePro software in each of five separate culture wells/condition. $\mathbf{b}$. SEMA4D inhibits process extension and migratory function of astrocytes; pepinemab reverses effects. Cell-free area in Radius 24-well Cell Migration Assay (Cell Biolabs) was determined following culture of purified astrocytes for the indicated time in the presence or absence of recombinant SEMA4D $(15 \mathrm{mcg} / \mathrm{ml})$, added at time 0 . SEMA4D antibody "VX15" or isotype control antibody "CTRL Ig" $(50 \mathrm{ug} / \mathrm{ml})$ was added at time $=20$ hours to determine whether the effect is reversible. Results in replicate wells $(n=6)$ at each time point are normalized to cell-free area at time 0 . Statistical significance was determined using two-way ANOVA. c. iPSC-derived human astrocytes were cultured in presence of rSEMA4D or control protein rEGFR. Antibody blocking effect was determined by incubation with rSEMA4D or control protein rEGFR $(5 \mathrm{ug} / \mathrm{ml})$, in presence/absence of anti-SEMA4D antibody/VX15 or isotype control human IgG4 antibody $(25 \mathrm{ug} / \mathrm{ml})$ for 48 hours. Morphologic changes in primary branch length and number of branches were quantified. Statistical significance was determined using Dunnet's multiple comparisons test, compared to control at each time point. ${ }^{*} p<0.05 ;{ }^{* *} p<0.01 ;{ }^{* * *} p<0.001 ;{ }^{* * * *} p<0.0001$. 


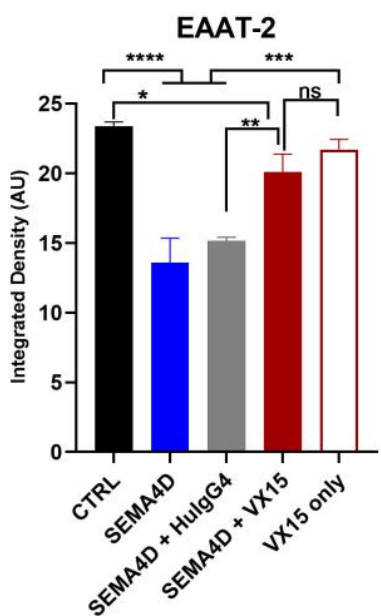

b

GLUT-1

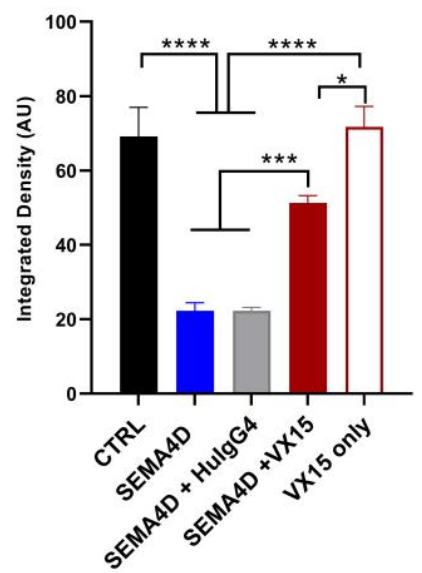

MCT-4
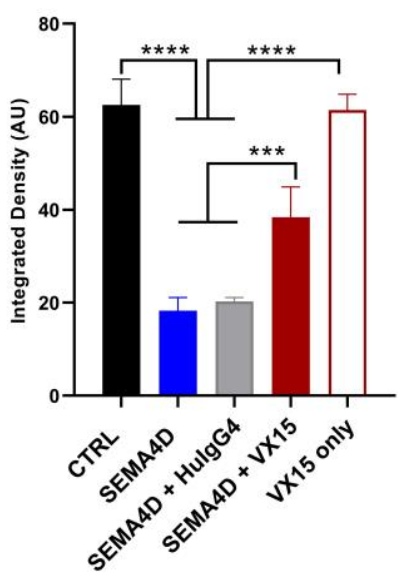
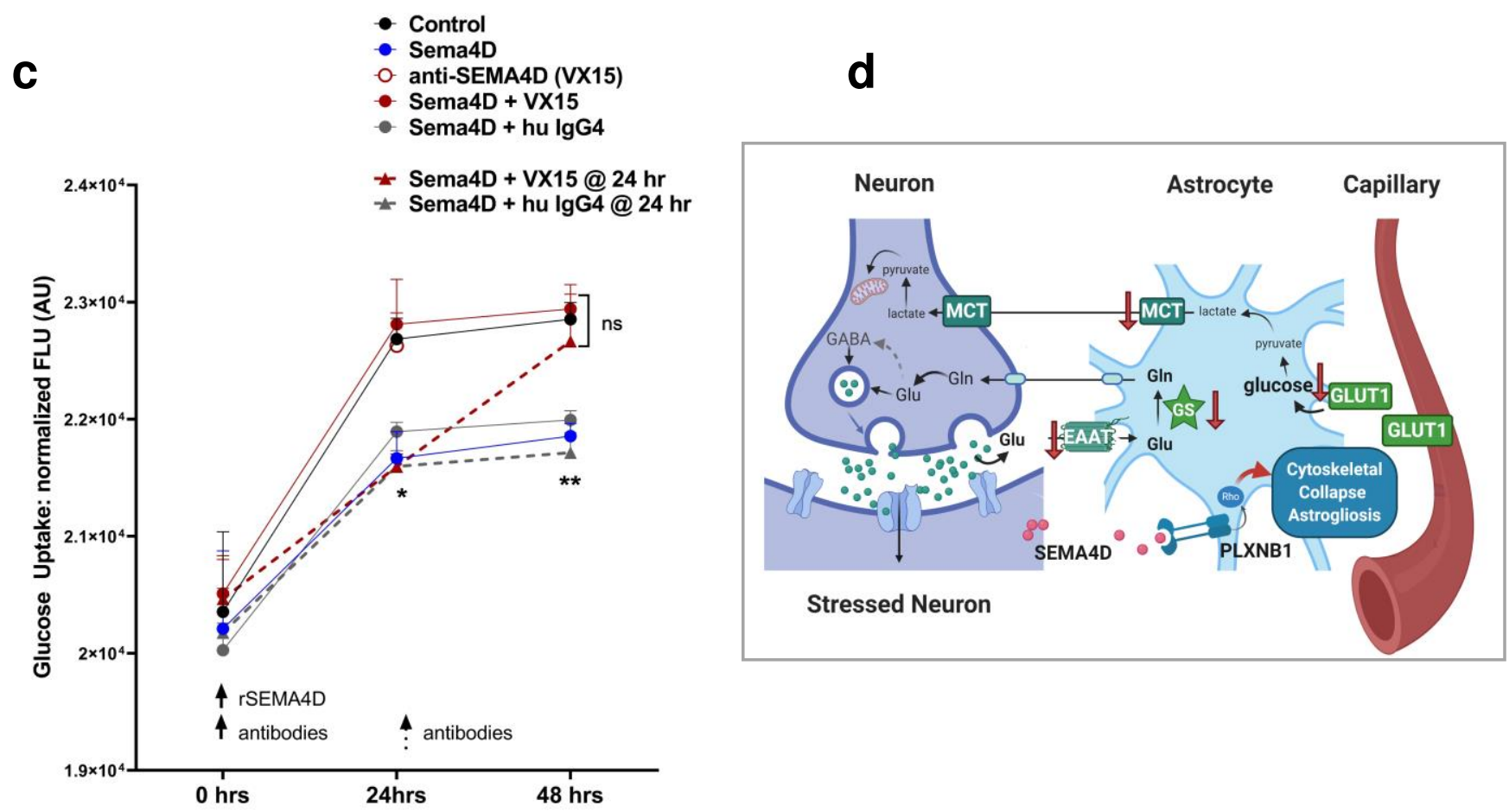

Figure 6: Astrocytic functions of glutamate and glucose transport are mediated by SEMA4D signaling and are reversible with antibody blockade of SEMA4D. iPSC-derived human astrocytes cultures were treated as in Figure 5 and stained for a. glutamate transporter (EAAT-2), and b. glucose (GLUT-1) and lactate (MCT-4) transporters. Antibody blockade of SEMA4D restored transporter expression. c. Glucose uptake was measured in human astrocyte cultures following incubation with rSEMA4D or control protein rEGFR $(25 \mathrm{ug} / \mathrm{ml})$, in presence/absence of anti-SEMA4D antibody/VX15 or isotype control human lgG4 antibody (125 ug/ml) in triplicate wells for 48 hours (circles). To evaluate reversal of activity, rSEMA4D was added at time 0 and antibodies were added at $\mathrm{t}=24 \mathrm{hr}$ (squares). Quantification for each condition is shown as average+SEM from 3 wells/condition/timepoint. Statistical significance was determined using Dunnet's multiple comparisons test, compared to control at each time point. ${ }^{*} p<0.05$; ${ }^{* *} p<0.01 ;{ }^{* * *} p<0.001 ;{ }^{* * *} p<0.0001$. d. Proposed SEMA4D effects on astrogliosis and neuronal support. SEMA4D is upregulated in stressed neurons and binds to PLXNB1 receptor to trigger reactive astrogliosis, characterized by morphologic reorganization of the cytoskeleton and retraction of dendritic processes, downregulation of glucose and lactate transporters (GLT1 and $\mathrm{MCT}$ ), downregulation of glutamate receptor (EAAT2), and reduction in glutamine synthetase (GS), key functional receptors and enzymes in astrocytes; activation induced changes indicated by red arrows.

Dysfunctional transport and conversion of metabolic and neurotransmitter substrates reduces astrocytic neuroprotection mechanisms and impairs recycling glutamate to glutamine in astrocytes and glutamine to glutamate and GABA in neurons. Astrocytic regulation of neuronal loss and reduced synaptic function contribute to pathogenic mechanisms in neurodegenerative disease. Image created with BioRender.com. 
a
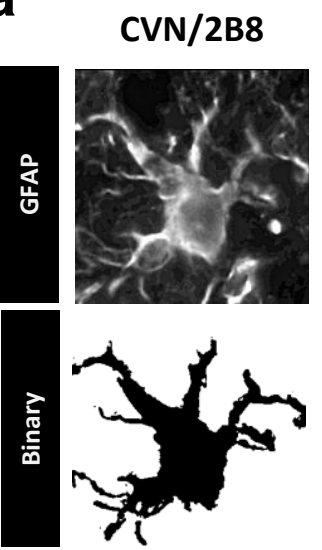

CVN/67
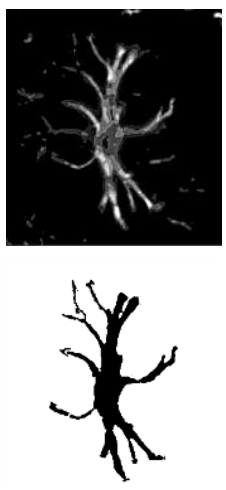

WT
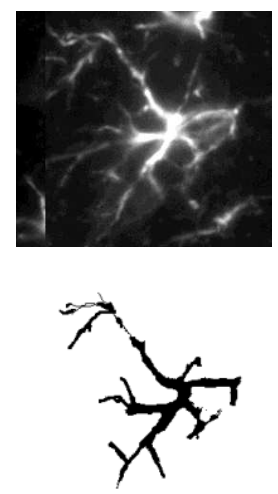

b

\section{Astrocyte Morphology}

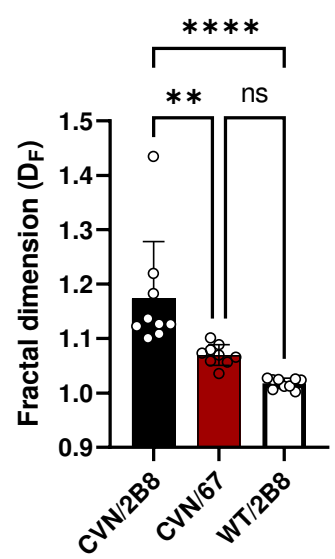

C

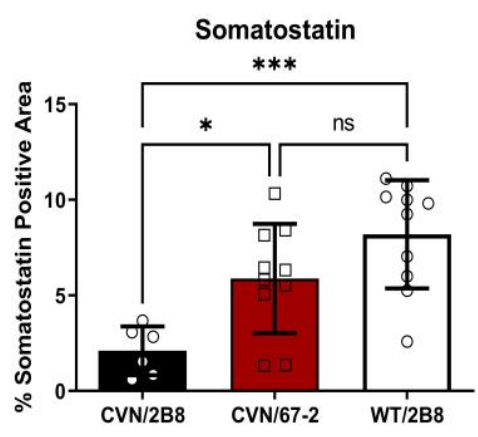

V-GLUT

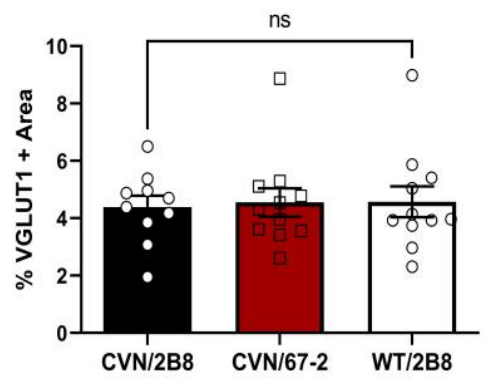

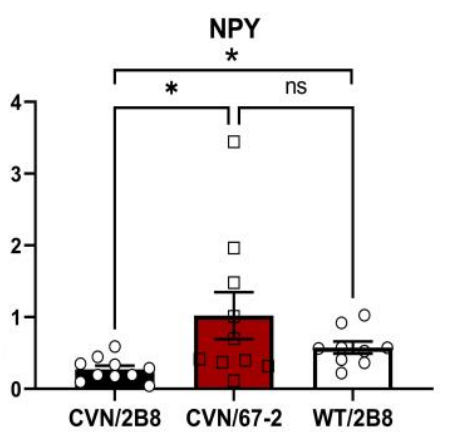

Synaptophysin

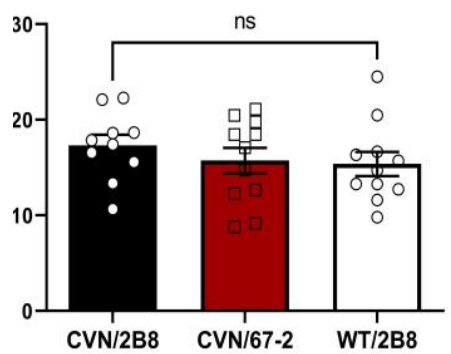

Figure 7: SEMA4D antibody treatment reverses astrocyte activation and synaptic function in AD mice. a. CA1 hippocampal region of CVN and wild type mice were stained GFAP and $\mathbf{b}$. fractal dimension analysis demonstrates significant changes in brains of CVN mice compared to wild type, which is restored following treatment with anti-SEMA4D antibody. c. The hippocampal region was stained with anti-somatostatin antibody or antiNeuropeptide-Y (NPY) to identify specific subsets of inhibitory neurons that begin to degenerate during early AD pathogenesis. No effects on excitatory synapses were observed in diseased mice (as determined by Synaptophysin and VGLUT-1 staining). Percentages were quantified for all animals ( $n=9-13 /$ group) and normalized to total area scanned; values for each mouse, along with group mean and standard error bars are shown. ${ }^{*} p<0.05$ and ${ }^{* * *} p<0.005$ by 1 -way ANOVA with Bonferroni's Multiple Comparison Test. 


\section{Figures}
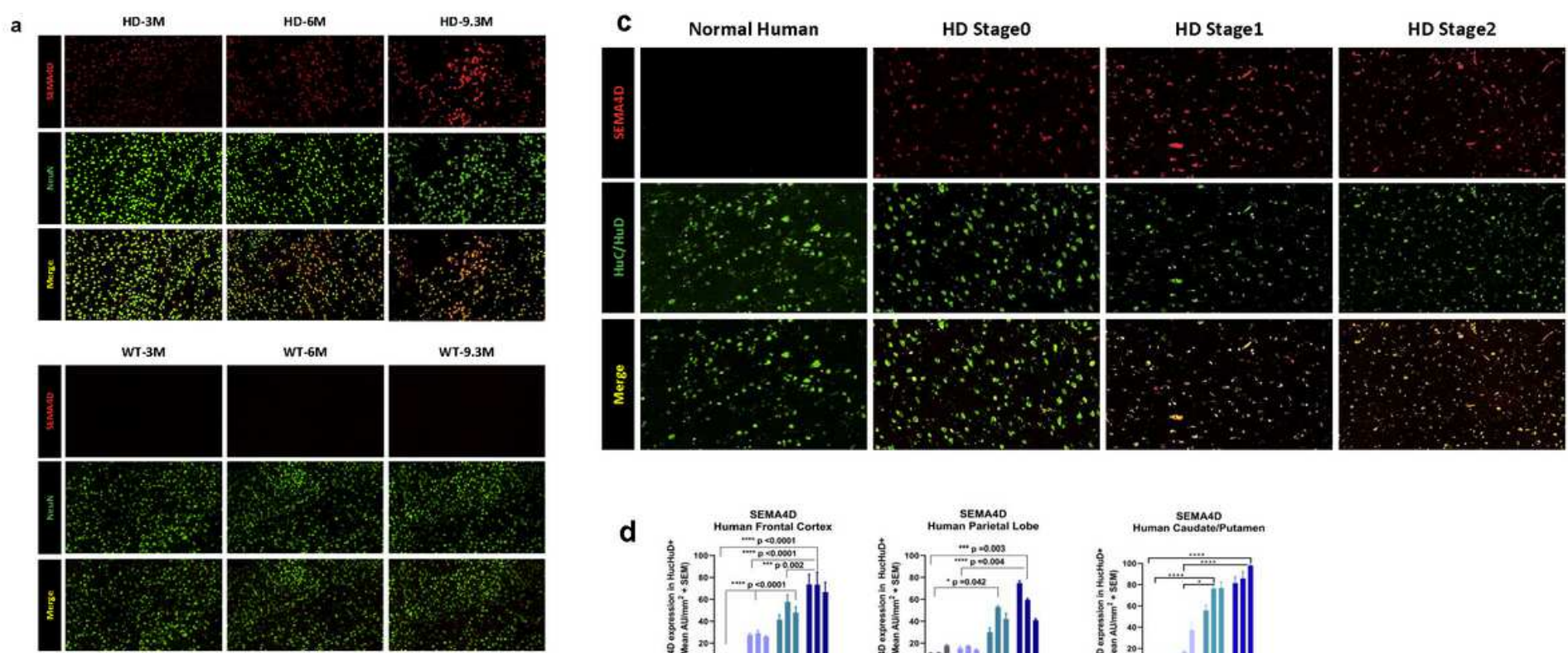

b
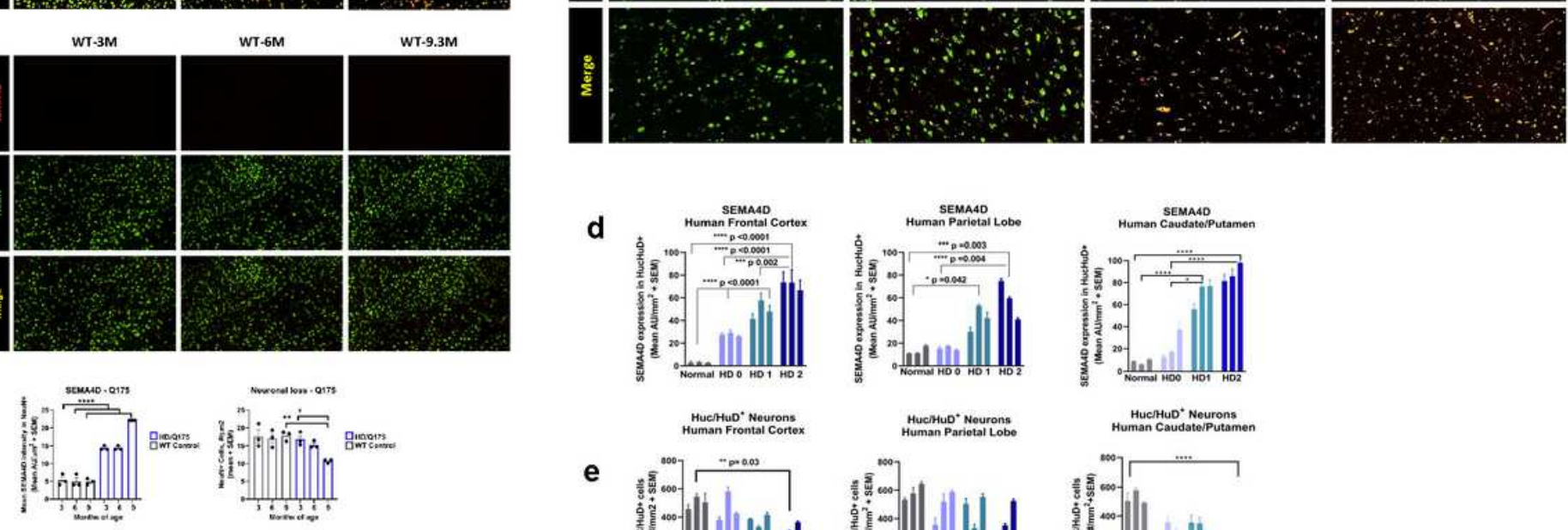

e
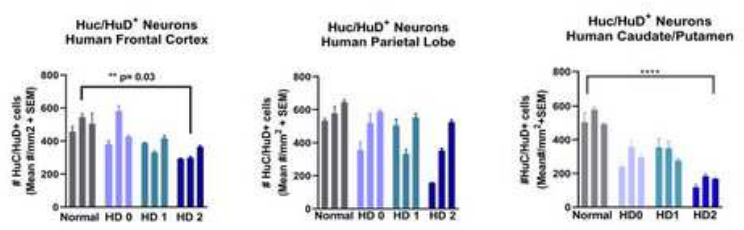

Figure 1

SEMA4D is increasingly upregulated in HD neurons in parallel with neuronal loss. a. Q175 knock-in mouse model of HD exhibits age-dependent upregulation and colocalization of SEMA4D in cortical neurons. b. Quantification of SEMA4D expression and number of neurons in Q175 model. Mean of entire coronal section +SEM (bar) with 3 mice/age group (closed circles). c. SEMA4D is increasingly upregulated with increasing human HD pathological stage in parallel with neuronal loss. Representative images of frontal lobe are shown. d. SEMA4D expression and e. number of neurons were quantified across entire human autopsy sections of frontal cortex (Inferior frontal gyrus, Brodmann area -BA 44-45), parietal lobe (Somatosensory cortex-Brodmann area- BA 1,2,3 and part of frontal cortex BA4) and striatum (caudate/putamen) regions. Mean of 3-4 consecutive sections +SEM is shown for 3 subjects/condition. Group differences and statistical significance was determined using one-way ANOVA

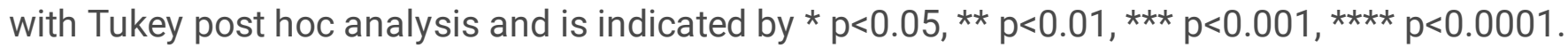


a
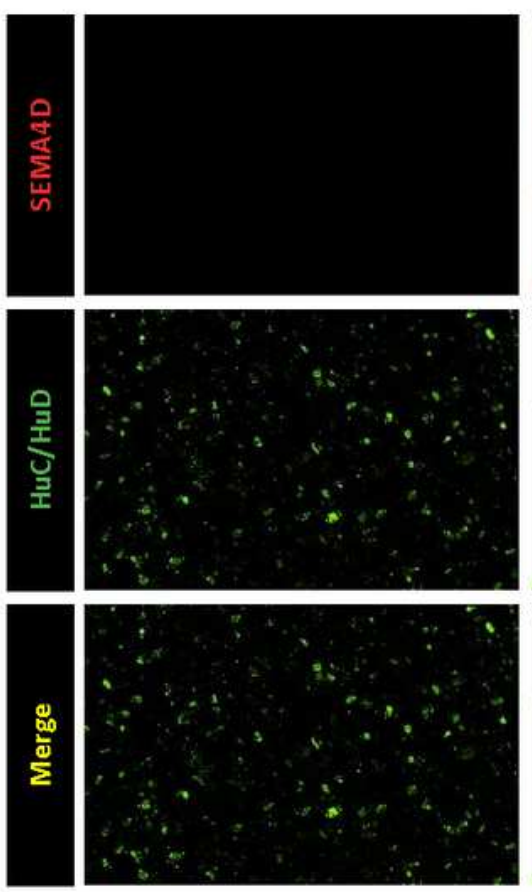

$A D$
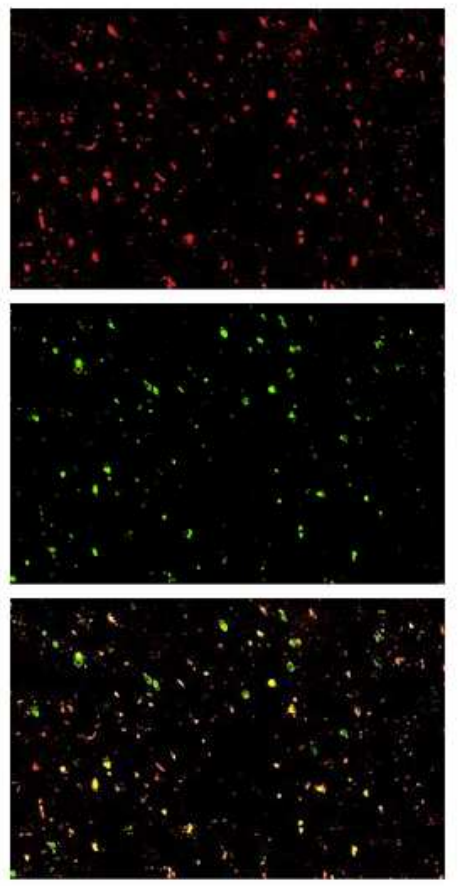

b Human Thalamus

Human Temporal Lobe

Human Frontal Cortex
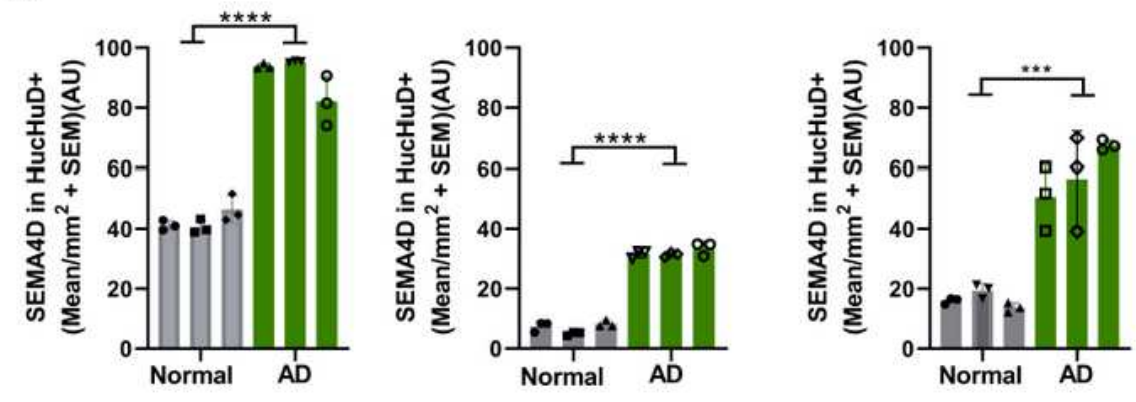

C Human Thalamus

Human Temporal Lobe

Human Frontal Cortex
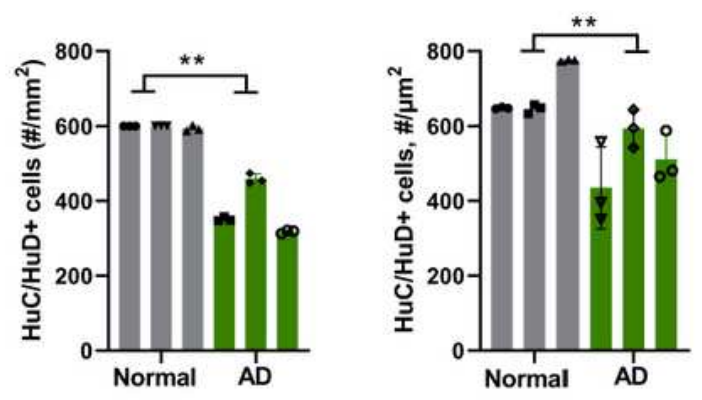

\section{Figure 2}

SEMA4D is upregulated in neurons of human AD. a. Representative images of human temporal lobe sections stained for SEMA4D and Huc/HuD(neuronal cell body). b. SEMA4D expression and c.number of neurons were quantified across entire human $A D$ autopsy sections of thalamus, temporal lobe (Brodmann area -BA 38), frontal cortex (Inferior frontal gyrus, Brodmann area -BA 44-45). Imaging of three consecutive slices of brain regions approximately $8 \mathrm{mmX10mm}$ in size was performed. Mean of 3-4 
consecutive sections +SEM is shown for 3 subjects/condition. Group differences and statistical significance was determined using one-way ANOVA with Tukey post hoc analysis and is indicated by * $p<0.05, * \star p<0.01, * \star \star p<0.001, * \star \star \star x<0.0001$.
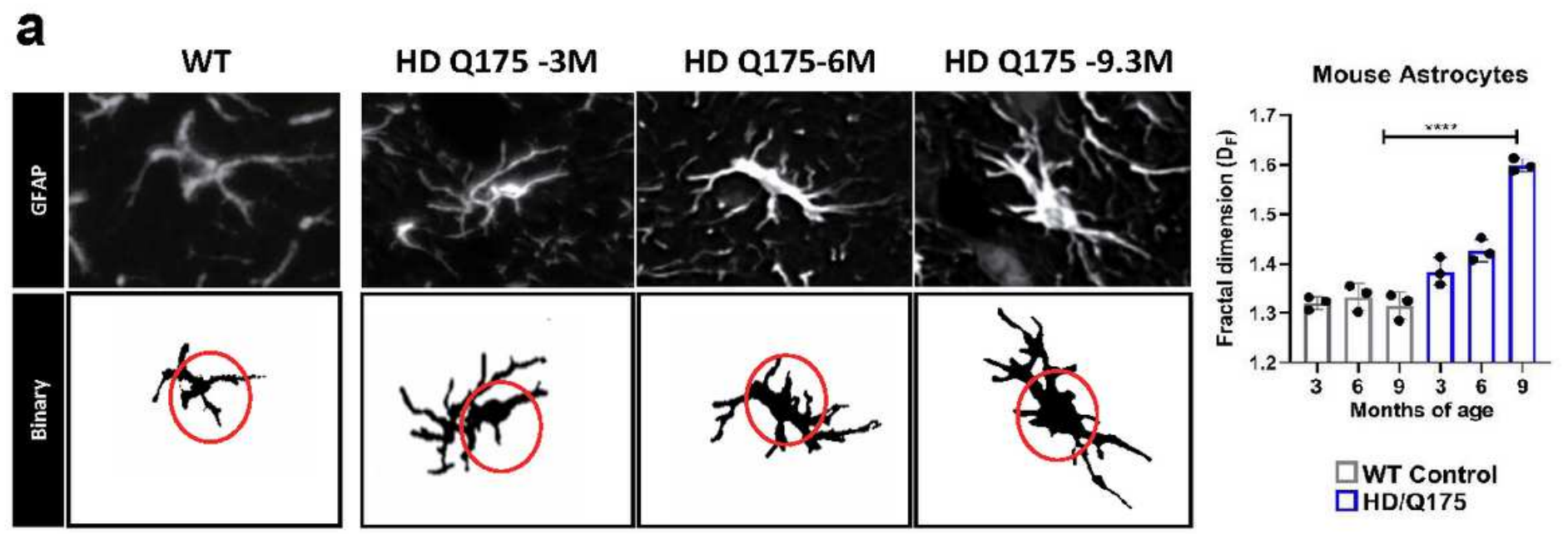

b

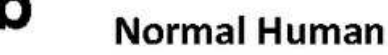

HD stage 0
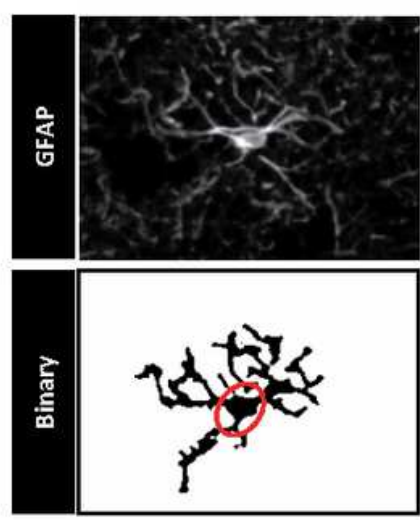
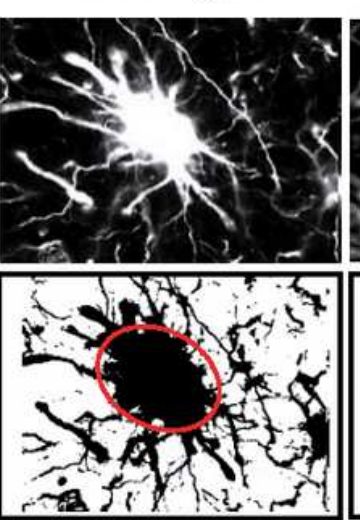

AD
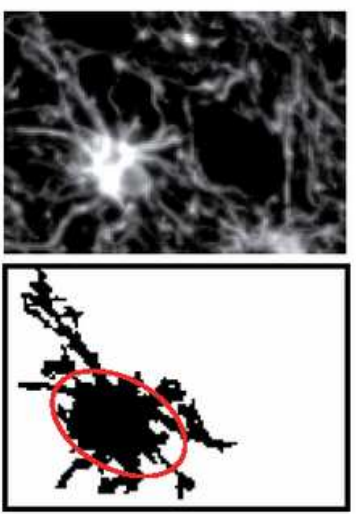

Human Astrocytes

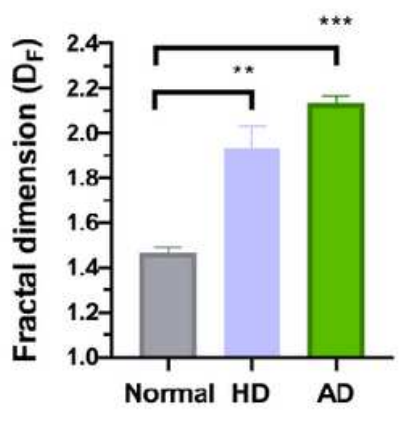

Figure 3

SEMA4D+ cells are in close proximity to astrocytes; morphologic changes indicative of astrogliosis are apparent with disease progression. a. GFAP staining of caudoputamen from analysis of 3 mice/timepoint are shown and were obtained from mouse brain coronal sections $(\sim 5 \mathrm{uM})$; representative images shown at 20X magnification. Fractal dimension analysis of astrocytes in mouse frontal cortex demonstrate significant changes in brains of Q175 mice at 9.3 months of age. b. Representative images of frontal lobe from non-diseased human, StageO HD and AD subjects are shown (20X magnification). Fractal dimension analysis of astrocytes in frontal cortex demonstrate significant changes between nondiseased ("normal") and HD or AD. 
a

Normal Human

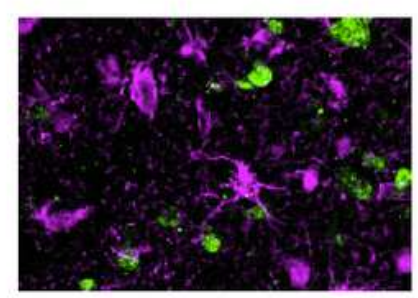

Huntington's Disease
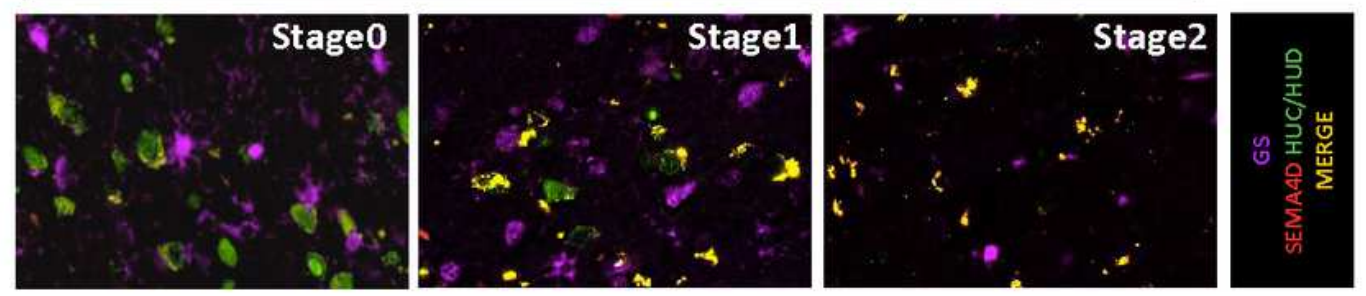

b
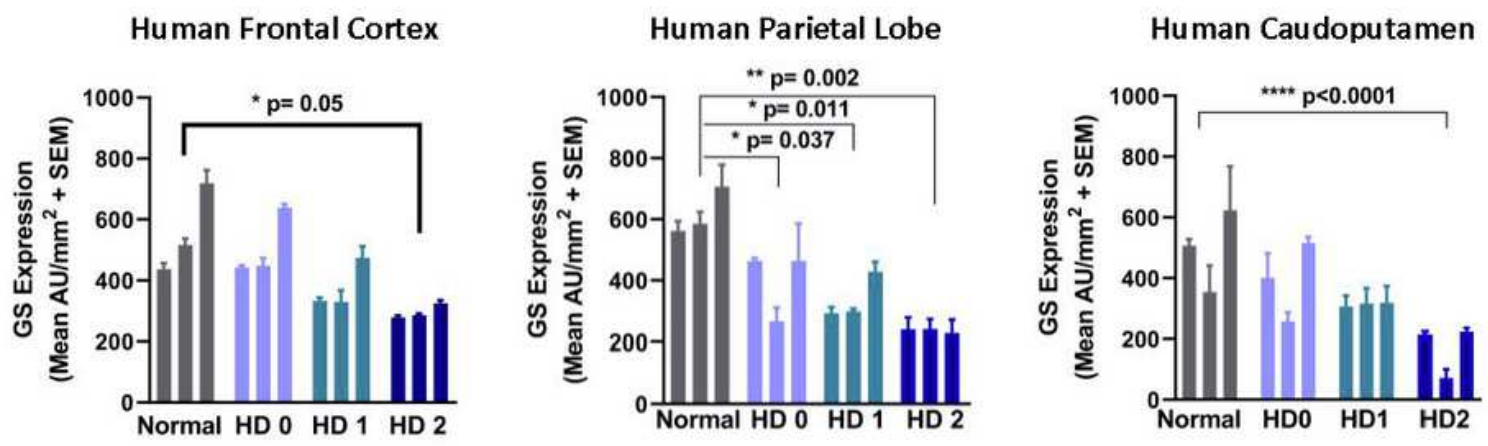

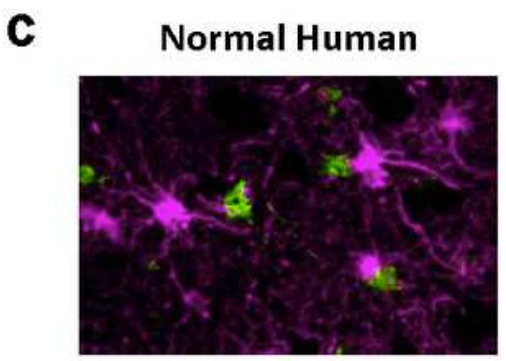

Human Thalamus

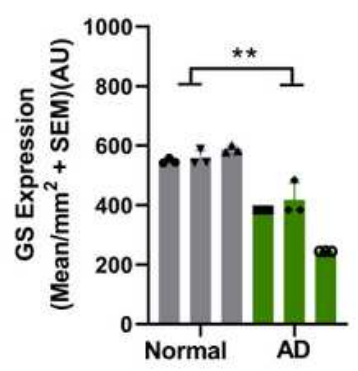

Alzheimer's Disease

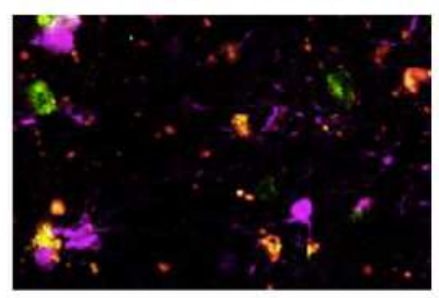

Human Temporal Lobe

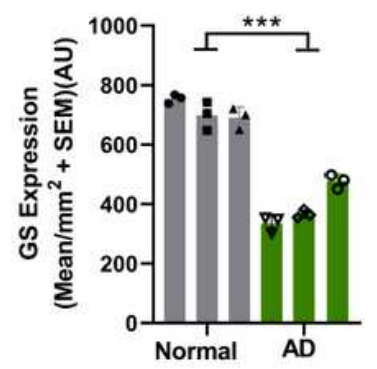

Human Frontal Cortex

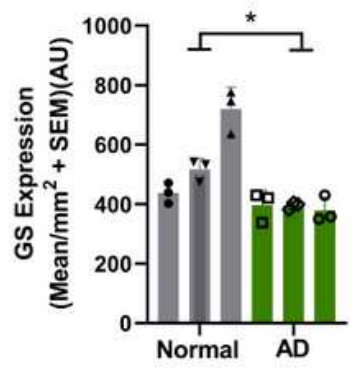

Figure 4

Downregulation of GS in reactive astrocytes is coincident with SEMA4D upregulation in neurons of HD and AD brains. Reactive astrocyte morphology, with reduced GS expression and retracted astrocytic endfeet, along with upregulation of neuronally expressed SEMA4D in HD $(a, b)$ and $A D(b, c) a$. Human frontal cortex sections were stained for glutamine synthetase (GS, astrocyte cell body and processes), SEMA4D, and Huc/HuD (neuronal cell body). b. Number of GS+ cells were quantified as described in Fig. 
1e. c. Human temporal lobe sections were stained for SEMA4D, and Huc/HuD, glutamine synthetase. d. Number of GS+ cells were quantified as described in Fig. 2c.

a

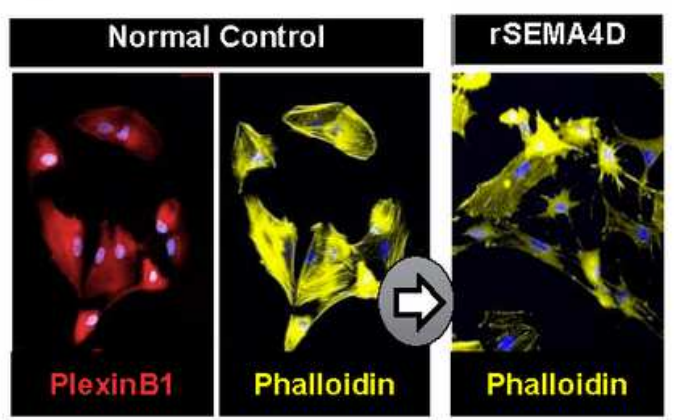

b
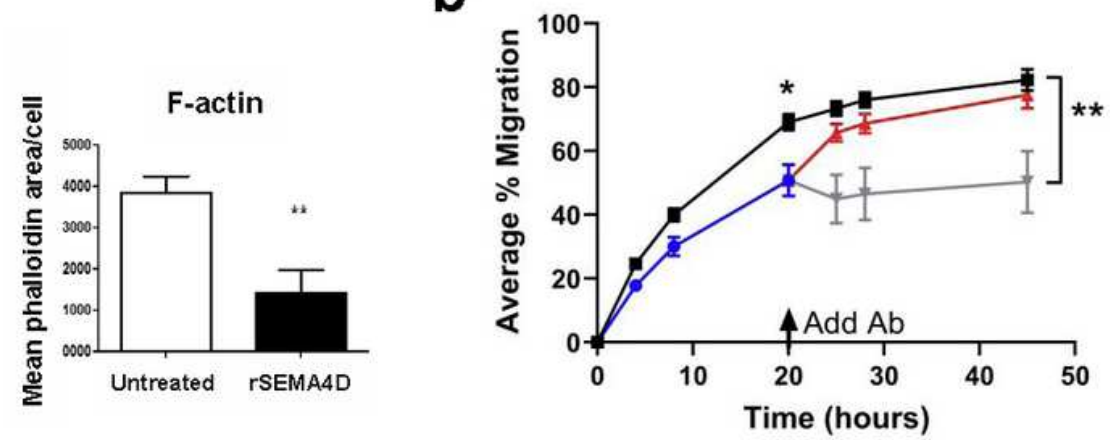

(hours)

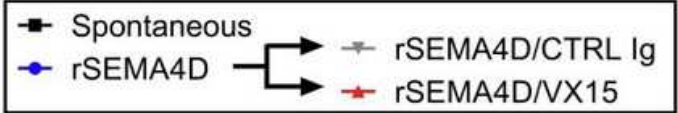

C

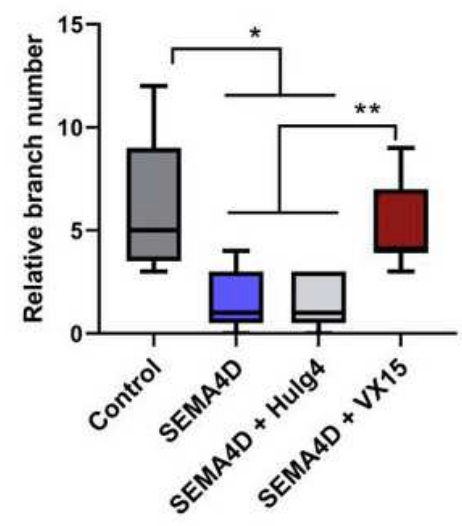

Branch number

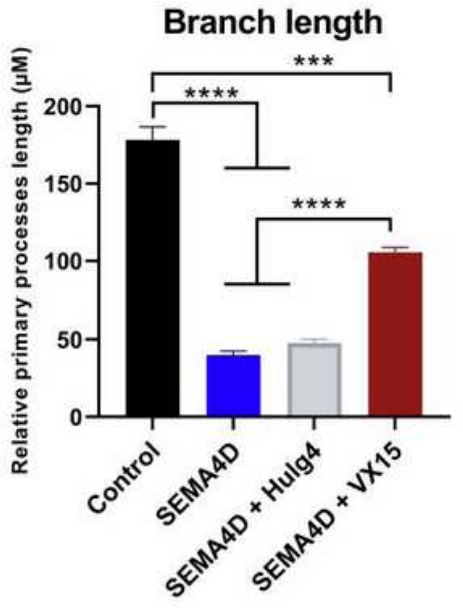

\section{Figure 5}

Reactive astrocyte morphologic changes are mediated by SEMA4D signaling through its receptor PlexinB1 and are reversible with antibody blockade of SEMA4D. a. Primary rat astrocyte cultures were stained for expression of receptor PLXNB1 (red), F-actin filaments (phalloidin, yellow) and nuclei (Dapi, blue) before (center) and after (right) one-hour treatment with recombinant SEMA4D. Representative images are shown. Mean phalloidin-positive area/cell in a field of $\sim 300$ cells was quantified using ImagePro software in each of five separate culture wells/condition. b. SEMA4D inhibits process extension and migratory function of astrocytes; pepinemab reverses effects. Cell-free area in Radius 24-well Cell Migration Assay (Cell Biolabs) was determined following culture of purified astrocytes for the indicated time in the presence or absence of recombinant SEMA4D $(15 \mathrm{meg} / \mathrm{ml})$, added at time 0 . SEMA4D antibody "VX15" or isotype control antibody "CTRL lg" (50 ug/ml) was added at time $=20$ hours to determine whether the effect is reversible. Results in replicate wells $(n=6)$ at each time point are normalized to cell-free area at time 0 . Statistical significance was determined using two-way ANOVA. c. iPSC-derived human astrocytes were cultured in presence of rSEMA4D or control protein rEGFR. Antibody blocking effect was determined by incubation with rSEMA4D or control protein rEGFR $(5 \mathrm{ug} / \mathrm{ml})$, in presence/absence of anti-SEMA4D antibody/VX15 or isotype control human lgG4 antibody $(25 \mathrm{ug} / \mathrm{ml})$ 
for 48 hours. Morphologic changes in primary branch length and number of branches were quantified. Statistical significance was determined using Dunnet's multiple comparisons test, compared to control at each time point. ${ }^{*} p<0.05 ;{ }^{*} p<0.01 ; * \star * p<0.001 ; * \star \star * p<0.0001$

a

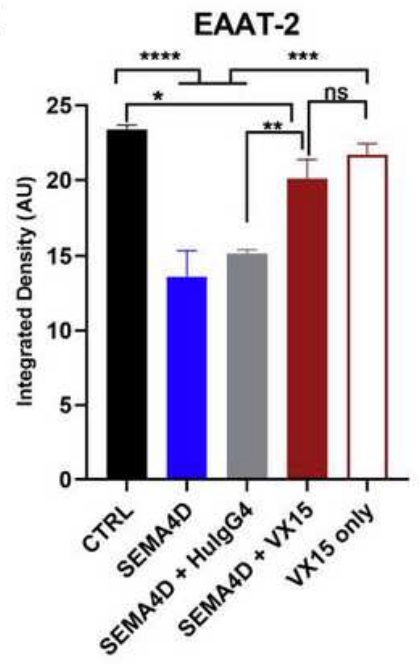

b

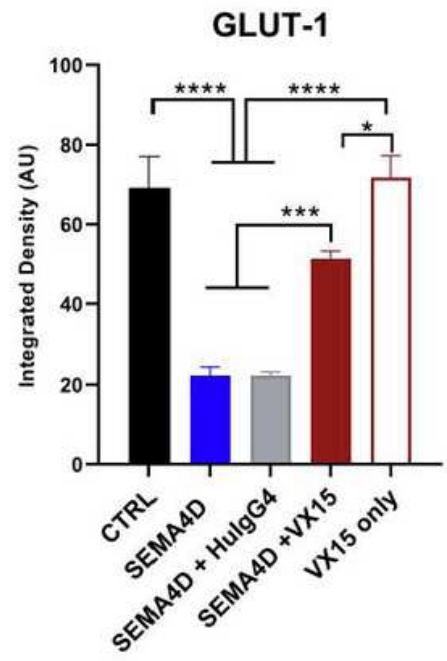

MCT-4

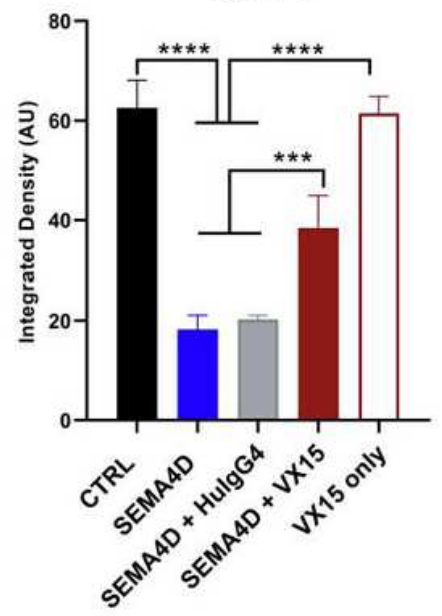

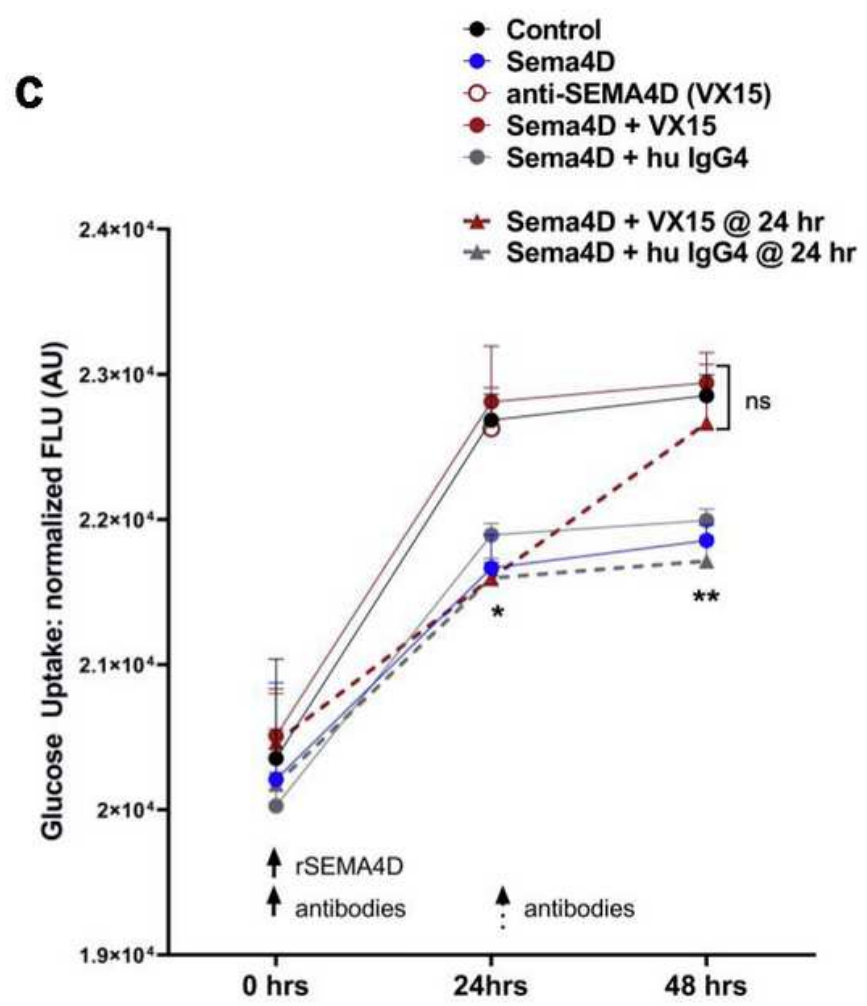

d

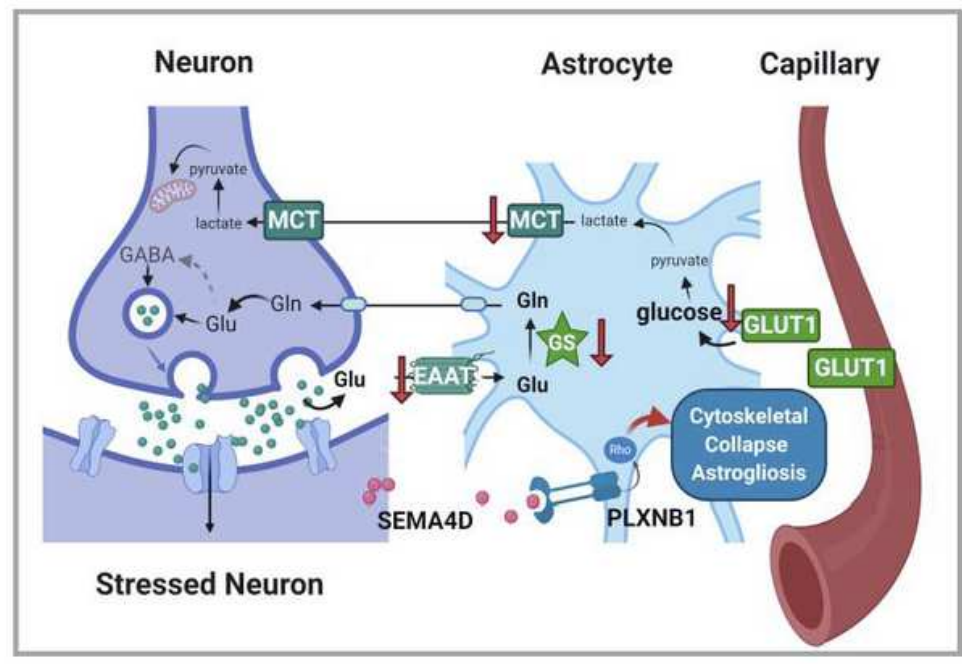

Figure 6

Astrocytic functions of glutamate and glucose transport are mediated by SEMA4D signaling and are reversible with antibody blockade of SEMA4D. iPSC-derived human astrocytes cultures were treated as in Figure 5 and stained for a. glutamate transporter (EAAT-2), and b. glucose (GLUT-1) and lactate (MCT-4) transporters. Antibody blockade of SEMA4D restored transporter expression. c. Glucose uptake was measured in human astrocyte cultures following incubation with rSEMA4D or control protein rEGFR (25 $\mathrm{ug} / \mathrm{ml}$ ), in presence/absence of anti-SEMA4D antibody/VX15 or isotype control human IgG4 antibody 
$(125 \mathrm{ug} / \mathrm{ml})$ in triplicate wells for 48 hours (circles). To evaluate reversal of activity, rSEMA4D was added at time 0 and antibodies were added at $\mathrm{t}=24 \mathrm{hr}$ (squares). Quantification for each condition is shown as average+SEM from 3 wells/condition/timepoint. Statistical significance was determined using Dunnet's multiple comparisons test, compared to control at each time point. ${ }^{*} \mathrm{p}<0.05 ;{ }^{* \star} \mathrm{p}<0.01 ;{ }^{* \star \star} \mathrm{p}<0.001$; $\star * \star \star p<0.0001$. d. Proposed SEMA4D effects on astrogliosis and neuronal support. SEMA4D is upregulated in stressed neurons and binds to PLXNB1 receptor to trigger reactive astrogliosis, characterized by morphologic reorganization of the cytoskeleton and retraction of dendritic processes, downregulation of glucose and lactate transporters (GLT1 and MCT), downregulation of glutamate receptor (EAAT2), and reduction in glutamine synthetase (GS), key functional receptors and enzymes in astrocytes; activation induced changes indicated by red arrows. Dysfunctional transport and conversion of metabolic and neurotransmitter substrates reduces astrocytic neuroprotection mechanisms and impairs recycling glutamate to glutamine in astrocytes and glutamine to glutamate and GABA in neurons. Astrocytic regulation of neuronal loss and reduced synaptic function contribute to pathogenic mechanisms in neurodegenerative disease. Image created with BioRender.com. 
a

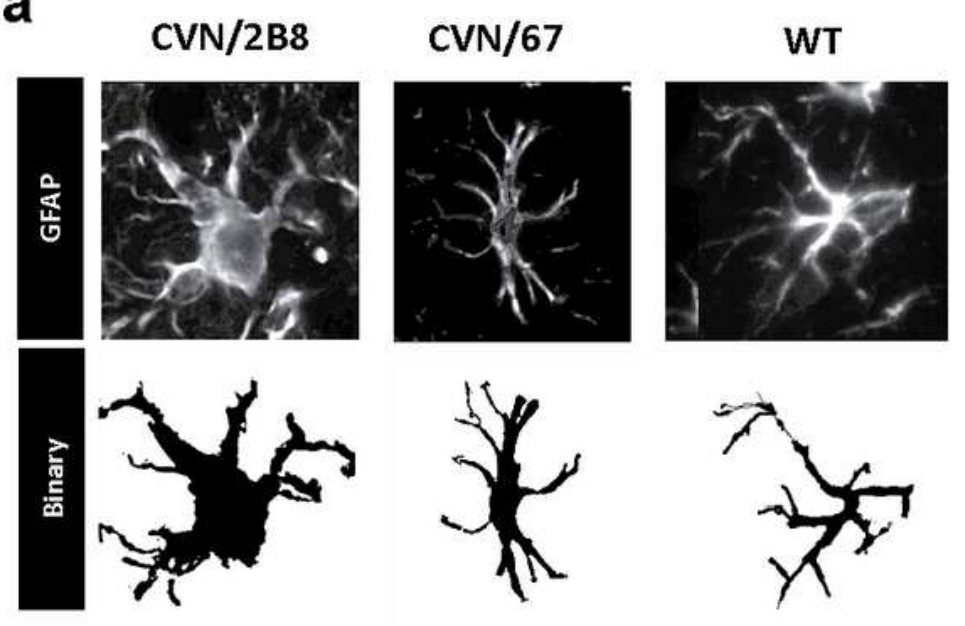

b

Astrocyte Morphology

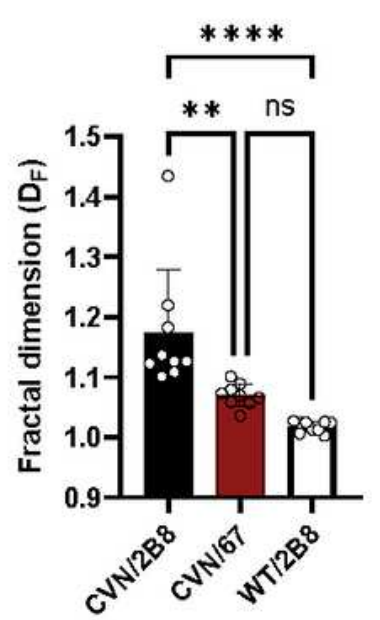

C
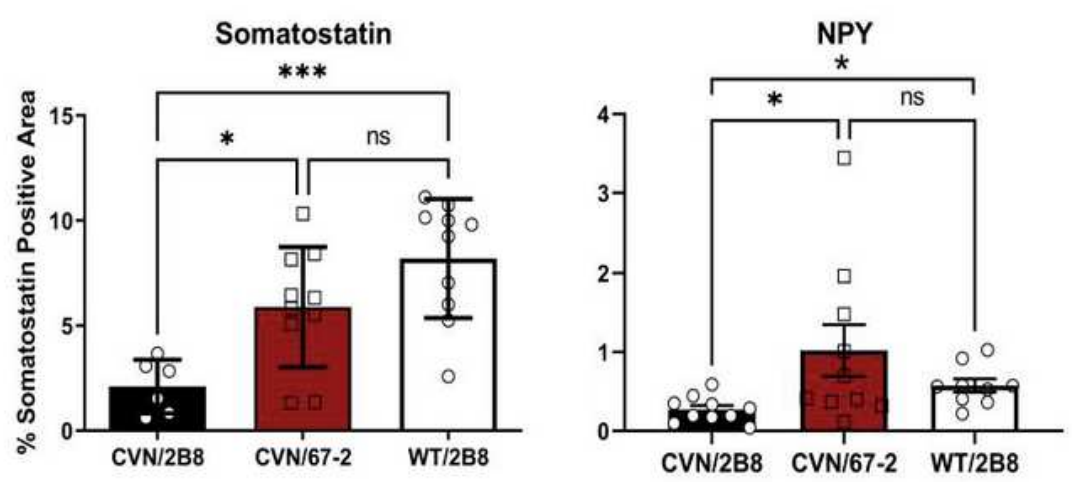

V-GLUT

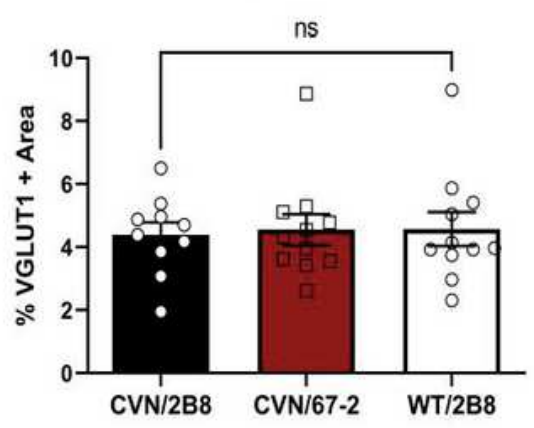

Synaptophysin

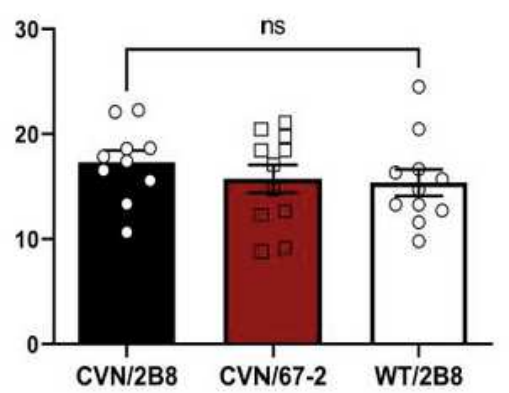

Figure 7

SEMA4D antibody treatment reverses astrocyte activation and synaptic function in AD mice. a. CA1 hippocampal region of $\mathrm{CVN}$ and wild type mice were stained GFAP and $b$. fractal dimension analysis demonstrates significant changes in brains of CVN mice compared to wild type, which is restored following treatment with anti-SEMA4D antibody. c. The hippocampal region was stained with antisomatostatin antibody or anti- Neuropeptide-Y (NPY) to identify specific subsets of inhibitory neurons 
that begin to degenerate during early $A D$ pathogenesis. No effects on excitatory synapses were observed in diseased mice (as determined by Synaptophysin and VGLUT-1 staining). Percentages were quantified for all animals ( $n=9-13 /$ group) and normalized to total area scanned; values for each mouse, along with group mean and standard error bars are shown. ${ }^{*} p<0.05$ and ${ }^{* *} \mathrm{p}<0.005$ by 1 -way ANOVA with Bonferroni's Multiple Comparison Test.

\section{Supplementary Files}

This is a list of supplementary files associated with this preprint. Click to download.

- ZaudererNatCommSUPPtablesandfigs.pdf 